\title{
The evolution of vibrational excitations in glassy systems
}

\author{
W. Götze and M.R. Mayr \\ Physik-Department, Technische Universität München, 85747 Garching, Germany
}

\begin{abstract}
The equations of the mode-coupling theory (MCT) for ideal liquid-glass transitions are used for a discussion of the evolution of the density-fluctuation spectra of glass-forming systems for frequencies within the dynamical window between the band of high-frequency motion and the band of low-frequencystructural-relaxation processes. It is shown that the strong interaction between density fluctuations with microscopic wave length and the arrested glass structure causes an anomalous-oscillation peak, which exhibits the properties of the so-called boson peak. It produces an elastic modulus which governs the hybridization of density fluctuations of mesoscopic wave length with the boson-peak oscillations. This leads to the existence of high-frequency sound with properties as found by $\mathrm{X}$-ray-scattering spectroscopy of glasses and glassy liquids. The results of the theory are demonstrated for a model of the hard-sphere system. It is also derived that certain schematic MCT models, whose spectra for the stiff-glass states can be expressed by elementary formulas, provide reasonable approximations for the solutions of the general MCT equations.
\end{abstract}

PACS numbers: 64.70.Pf, 63.50.+x, 61.20.Lc 


\section{INTRODUCTION}

The excitation spectra of normal liquids are located in the $\mathrm{THz}$ band. But if the liquid can be supercooled new spectra evolve within the $\mathrm{GHz}$ band as precursors of the glass transition. These spectra are due to structural-relaxation processes. In addition there appear vibrational spectra for frequencies $\omega$ above $0.1 \mathrm{THz}$ which are characteristic for glasses and glass-forming liquids, namely high-frequency sound and the so-called boson peak. In this paper a microscopic theory shall be presented for these vibrational excitations.

High-frequency sound was discovered only recently by $\mathrm{X}$-ray-scattering spectroscopy in various glass-forming systems: in aqueous solutions of $\mathrm{LiCl}$ [1], glycerol [1, 2], silica [3] 6], orthoterphenyl [2,7], $0.4 \mathrm{Ca}\left(\mathrm{NO}_{3}\right)_{2}$ 0.6 $\mathrm{KNO}_{3}$ [8], and $\mathrm{B}_{2} \mathrm{O}_{3}$ [8]. Molecular-dynamics simulations identified these modes for models of argon glass [9, 10], $\mathrm{ZnCl}_{2}$ [11], and silica [12,13]. Five features of high-frequency sound can be inferred from the cited work. First, the resonance position $\Omega_{q}^{\max }$ of the dynamical structure factor $S_{q}(\omega)$ is a linear function of the wave number $q, \Omega_{q}^{\max }=v_{\infty} q$, and this for $q$ as large as 4 to $8 \mathrm{~nm}^{-1}$. Here $v_{\infty}$ is the same high-frequency sound speed as known from Brillouin-scattering spectroscopy done for wave vectors which are about two orders of magnitude smaller. Second, the resonance width $\Gamma_{q}$ exhibits a quadratic wave-vector dependence: $\Gamma_{q}=\gamma q^{2}$. Hence density fluctuations propagate as known from the theory of elastic media for wave lengths down to the order of the inter-particle distances [1]. This holds with the reservation that the spectrometer resolution of about $0.4 \mathrm{THz}$ causes considerable uncertainties in the data for $\Omega_{q}^{\max }$ and $\Gamma_{q}$ and that it is difficult to separate the inelastic-scattering signal from the huge background due to the resolution-broadened quasi-elastic scattering. The damping of ordinary sound in crystals or liquids depends strongly on temperature. As a third remarkable feature of high-frequency sound one observes that $\Gamma_{q}$ exhibits only a weak temperature dependence. For silica and orthoterphenyl no temperature dependence of $\Gamma_{q}$ could be detected even though $\Omega_{q}^{\max }$ decreases with increasing temperature considerably [2, 4, 5, [7]. For hydrodynamic sound the resonances exhaust the inelastic spectrum of the density fluctuations. Contrary to this one 
infers from the simulation work [9, 10,13] as a fourth property of high-frequency sound that the resonances are superimposed on a broad intense background spectrum of $S_{q}(\omega)$. As a fifth property one finds for the simulation results of silica [13] that the background spectrum exhibits a low-frequency threshold. This conclusion is supported by the measurement of the X-ray-scattering spectrum of densified silica [6], which demonstrates that there is a suppression of $S_{q}(\omega)$ for small $\omega$ relative to the hydrodynamics description for the dynamical structure factor.

The so-called boson peak has been known for quite some time since it can be detected in the spectra obtained by standard techniques. It was measured for all the above cited systems, for example by Raman scattering for $\mathrm{LiCl}$ [14] and by neutron scattering for silica [3,15]. It was found by molecular-dynamics simulations for $\mathrm{ZnCl}_{2}$ [16] and silica [17,18]. Six features are typical for these peaks. First of all, the peaks are due to soft excitations. The position $\omega_{P}$ of the peak maximum is several times smaller than the Debye frequency $\omega_{D}$ of the system. In silica the peak causes an enhancement of the so-called density of states by a factor of about seven relative to the Debye spectrum [15]. Second, the anomalous peak is due to quasi-harmonic oscillations. Originally, this was concluded indirectly, since this assumption can explain the enhancement of the specific heat above Debye's $T^{3}$-law for temperatures $T$ near 10K [15]. Normal-mode analysis for a model of silica showed this result explicitly [17]. Third, the peak is skewed. There seems to be a low-frequency threshold $\Omega_{-}$so that the high-frequency wing of the peak extends further than the one on the low-frequency side [3, 14, 18]. Fourth, the peak appears to be related to some instability of the system since $\omega_{P}$ and $\Omega_{-}$decrease to zero upon heating the system towards some characteristic temperature $T_{b p}[14,18]$. The mentioned four features are also exhibited by the density of states of a harmonic lattice with a random distribution of force constants [19]. As a fifth property one observes that a quasi-elastic relaxation peak appears for $T$ increasing towards $T_{b p}$ which eventually buries the peak 14. Sixth, the peak position $\omega_{P}$ of the dynamical structure factor depends only weakly on wave vector $q$ if at all [3, 18,.

The discussions of this paper are done within the framework of the mode-coupling theory 
(MCT) for the evolution of glassy dynamics in simple systems. This theory is based on closed microscopic equations of motion for the density-correlation functions [20,21]. Previous work done in this context focused on elucidating the properties of the structural-relaxation phenomena, as described in Refs. [22,23] and the papers quoted there. In the following it will be shown that MCT also implies a theory for the evolution of anomalous-oscillation peaks (AOP) with the same six properties specified in the preceding paragraph for the so- $^{-}$ called boson peak. Furthermore it will be demonstrated that the AOP manifests itself in resonances of the density-fluctuation spectra for wave-vectors up to half the value of the Debye vector $q_{D}$, which exhibit the five features listed above for high-frequency sound.

MCT deals with states of matter for which the structure factor $S_{q}$ depends smoothly on wave vector $q$ and on control parameters like the temperature $T$ and density $\rho$. The equations of motion of the basic version of this theory, which will be used in this paper, exhibit a bifurcation from ergodic liquid dynamics to non-ergodic glass dynamics if $T$ or $\rho$ cross critical values $T_{c}$ or $\rho_{c}$ respectively. This bifurcation provides a model for an ideal liquid-glass transition. In the extended version of MCT the singular transition is replaced by regular crossover [23]. The crossover is connected with the evolution of structural relaxation. Extensive tests of the MCT predictions have been performed in recent years. Let us only mention one group of such tests which are of particular relevance for the following discussions. The MCT results for the Debye-Waller factors $f_{q}$ of the glass depend non trivially on $q$ and on $T$ or $\rho$. The results calculated for the hard-sphere system [20] agree with the data measured for hard-sphere colloids [24]. Similarly, the $f_{q}$ calculated for a Lennard-Jones mixture 25] agree within 10\% with the values deduced from molecular-dynamics studies [26]. These assessments of the theory and many other tests, which are reviewed in Ref. [27], show that MCT describes properly some essential features of structural relaxation. Since the MCT equations are rather involved most of the original work was done first for schematic models. These are truncations of the large set of equations to sets dealing with a few correlators only. The truncations are done with the intention to reduce the complexity of the mathematical machinery without loosing the essence of the features to be analyzed 
for the solution. It was noticed for schematic models dealing with a single correlator that the spectra for the states for $T \ll T_{c}$ or $\rho \gg \rho_{c}$ exhibit a broad peak, which is due to a superposition of harmonic oscillator spectra [22,28]. Tao et al. recognized that the evolution of these peaks due to changes of control parameters was similar to what they had measured for the boson peak of glassy aqueous $\mathrm{LiCl}$ [14]. Therefore they concluded that MCT implies a theory for the boson-peak spectrum. This conclusion was corroborated by a series of studies, where solutions of schematic models were used to describe quantitatively spectra of glassy liquids 29 34. The fits described structural-relaxation spectra in windows of several orders of magnitude in size in addition to parts of the boson-peak spectra for high frequencies. In the following this earlier work will be extended to a systematic theory for the general MCT equations. This theory implies, in particular, the suggestion that the characteristic temperature $T_{b p}$ for the boson-peak dynamics is identical with $T_{c}$. Our results will be demonstrated comprehensively for a model of the hard-sphere system (HSS).

The paper is organized as follows. In Sec.II the basic equations for our calculations are formulated and the details for the HSS work are specified. The evolution of structural relaxation will be demonstrated in order to put the discussion in the proper context with the theory of the glass transition. Then, in Sec.III, the results for the evolution of the anomalous-oscillation peak and for high-frequency sound are presented. These oscillation features of the MCT dynamics are described within a generalized-hydrodynamics approach. In Sec.IV the phenomena are explained within a schematic-model analysis. In the concluding Sec.V our findings are discussed.

\section{BASIC EQUATIONS}

\section{A. A mode-coupling-theory model for a dense simple system}

The basic quantity specifying the equilibrium structure of a simple system is the structure

factor $S_{q}=\left\langle\left|\rho_{\vec{q}}\right|^{2}\right\rangle$, which is the canonical average of the squared modulus of the density 
fluctuations of wave vector $\vec{q}: \rho_{\vec{q}}=\sum_{\alpha} \exp \left(i \vec{q}_{\alpha}\right) / \sqrt{N}$. Here $\alpha$ labels the $\mathrm{N}$ particles at positions $\vec{r}_{\alpha}$ in the system of density $\rho$. The structure factor of amorphous systems depends on the wave vector modulus $q=|\vec{q}|$ only and it is usually expressed in terms of the direct correlation function $c_{q}: S_{q}=1 /\left(1-\rho c_{q}\right)$ [35]. The most relevant variables describing the dynamics of structure changes as function of time $t$ are the density correlators $\phi_{q}(t)=$ $\left\langle\rho_{\vec{q}}^{*}(t) \rho_{\vec{q}}\right\rangle / S_{q}$. The short time asymptote of these functions is specified by a characteristic frequency $\Omega_{q}: \phi_{q}(t)=1-\frac{1}{2}\left(\Omega_{q} t\right)^{2}+\cdots$. In the small-wave-vector limit one gets the dispersion law for sound; $\Omega_{q}=v_{0} q+O\left(q^{3}\right)$, where $v_{0}$ denotes the isothermal sound speed. For general wave vectors one obtains $\Omega_{q}^{2}=v^{2} q^{2} / S_{q}$, where $v$ denotes the thermal velocity of the particles [35]. In particular $v_{0}^{2}=v^{2} / S_{0}$. Within the Zwanzig-Mori formalism one can derive the exact equation of motion

$$
\ddot{\phi}_{q}(t)+\Omega_{q}^{2} \phi_{q}(t)+\Omega_{q}^{2} \int_{0}^{t} m_{q}\left(t-t^{\prime}\right) \dot{\phi}_{q}\left(t^{\prime}\right) d t^{\prime}=0 .
$$

The relaxation kernel $m_{q}(t)$ is a correlation function of fluctuating forces [35]. Let us introduce here and in the following Fourier-Laplace transformations to map $\phi_{q}, m_{q}$ and similar functions from the time domain onto the frequency domain according to the convention $\phi_{q}(\omega)=i \int_{0}^{\infty} \exp (i \omega t) \phi_{q}(t) d t=\phi_{q}^{\prime}(\omega)+i \phi_{q}^{\prime \prime}(\omega)$. The reactive part $\phi_{q}^{\prime}(\omega)$ and the dissipative part or the spectrum $\phi_{q}^{\prime \prime}(\omega)$ are connected via a Kramers-Kronig relation. Equation (10) is equivalent to the representation $\phi_{q}(\omega)=-1 /\left[\omega-\Omega_{q}^{2} /\left[\omega+\Omega_{q}^{2} m_{q}(\omega)\right]\right]$. The fluctuation dissipation theorem connects $\phi_{q}(\omega)$ with the dynamical susceptibility $\chi_{q}(\omega): \chi_{q}(\omega)=$ $\left[\omega \phi_{q}(\omega)+1\right] \chi_{q}^{T}$. Here $\chi_{q}^{T}=S_{q} /\left(\rho \mu v^{2}\right)$, with $\mu$ denoting the mass of the particles, is the isothermal compressibility [35]. Therefore Eq. (1a) is equivalent to

$$
\chi_{q}(\omega) / \chi_{q}^{T}=-\Omega_{q}^{2} /\left[\omega^{2}-\Omega_{q}^{2}+\Omega_{q}^{2} \omega m_{q}(\omega)\right] .
$$

Within the MCT kernel $m_{q}(t)$ is written as the sum of a regular contribution and a mode-coupling contribution, describing the cage effect of dense systems within Kawasaki's factorization approximation. In this paper we neglect the regular term. Hence one gets [20]: 


$$
m_{q}(t)=\mathcal{F}_{q}[\phi(t)]
$$

where the mode-coupling functional $\mathcal{F}_{q}$, considered as functional of the dummy variable $\tilde{f}$, is: $\mathcal{F}_{q}[\tilde{f}]=\sum_{\vec{k}+\vec{p}=\vec{q}} V(\vec{q}, \vec{k} \vec{p}) \tilde{f}_{k} \tilde{f}_{p}$. The coupling coefficients $V(\vec{q}, \vec{k} \vec{p})$ are determined by the equilibrium structure:

$$
V(\vec{q}, \vec{k} \vec{p})=\rho S_{q} S_{k} S_{p}\left[\vec{q}\left(\vec{k} c_{k}+\vec{p} c_{p}\right)\right]^{2} /\left(2 q^{4}\right)
$$

Let us approximate wave-vector integrals by Riemann sums, obtained by choosing an equally-spaced wave-vector grid of $\mathrm{M}$ terms. Thus the wave-vector index $q$ will be understood as a label running from 1 to $\mathrm{M}$. Correlators $\phi(t)$, kernels etc. are considered as M-component vectors. After this discretization the functional is a quadratic polynomial

$$
\mathcal{F}_{q}[\tilde{f}]=\sum_{k p=1}^{M} V_{q, k p} \tilde{f}_{k} \tilde{f}_{p}
$$

The positive coefficients $V_{q, k p}$ are related trivially to $V(\vec{q}, \vec{k} \vec{p})$ in Eq. (2b); for details the reader is referred to Ref. 36].

Equations (11) and (2) are closed. They define a unique solution for all parameters $\Omega_{q}>0, V_{q, k p} \geq 0$. The solution has all the properties of a correlator, i.e. the $\phi_{q}(t)$ are positive-definite functions given by non-negative spectra $\phi_{q}^{\prime \prime}(\omega)$. And the solutions depend smoothly on $\Omega_{q}$ and $V_{q, k p}$ for all finite time intervals [37,38]. Thus, Eqs. (11) and (22) formulate a well-defined model for a dynamics. The model is related to the physics of liquids by specifying $\Omega_{q}$ and $V_{q, k p}$ in terms of particle interactions and the control parameters $\rho, T$.

In the following the results will be demonstrated for the hard-sphere system (HSS). The structure factor is independent of $\mathrm{T}$ in this case, so that the only non-trivial control parameter is the packing fraction $\varphi$ of the spheres of diameter $d: \varphi=\pi d^{3} \rho / 6$. The structure factor $S_{q}$ is evaluated in the Percus-Yevick approximation [35]. Wave vectors will be considered up to a cutoff value $q^{*}=40 / d$ and they are discretized to $M=300$ values. The sphere diameter will be chosen as the unit of length, $d=1$, and the unit of time will be chosen such that the thermal velocity is $v=2.5$. 


\section{B. Ideal glass states}

The specified model exhibits a fold bifurcation [22]. For small coupling constants $V_{q, k p}$ the correlators $\phi_{q}(t)$ and kernels $m_{q}(t)$ decay sufficiently fast to zero for times tending to infinity so that the spectra $\phi_{q}^{\prime \prime}(\omega)$ and $m_{q}^{\prime \prime}(\omega)$ are continuous in $\omega$. Density fluctuations, which are created at time $t=0$, disappear for long times; and the same holds for the force fluctuations. The system approaches equilibrium for long times as expected for an ergodic liquid. This implies $\lim _{\omega \rightarrow 0} \omega m_{q}(\omega)=0$ and one concludes from Eq. (11b) that the static susceptibility $\hat{\chi}_{q}=\chi_{q}(\omega \rightarrow 0)$ agrees with the thermodynamic susceptibility, $\hat{\chi}_{q}=\chi_{q}^{T}$. For large coupling constants, on the other hand, there is arrest of density fluctuations for long times: $\phi_{q}(t \rightarrow \infty)=f_{q} ; 0<f_{q}<1$. Thus the perturbed system does not return to the equilibrium state. Similarly, there is arrest of the force fluctuations: $m_{q}(t \rightarrow \infty)=C_{q}>0$. The numbers $f_{q}, C_{q}$ are connected by the equations [20]:

$$
f_{q}=C_{q} /\left(1+C_{q}\right) \quad, \quad C_{q}=\mathcal{F}_{q}[f] \quad, \quad q=1,2, \ldots, M
$$

For this strong coupling solution the kernel exhibits a zero-frequency pole, $\lim _{\omega \rightarrow 0} \omega m_{q}(\omega)=$ $-C_{q}$, and therefore one concludes from Eq. (15) that the static susceptibility is smaller

than the thermodynamic one, $\hat{\chi}_{q}<\chi_{q}^{T}$, since $\hat{\chi}_{q}=\chi_{q}^{T} /\left(1+C_{q}\right)$. This is a signature for a non-ergodic state [39]. The system reacts more stiffly than expected for a canonical averaging. The dynamical structure factor $S_{q}(\omega)=S_{q} \phi_{q}^{\prime \prime}(\omega)$ exhibits a strictly elastic peak: $S_{q}(\omega)=\pi S_{q} f_{q} \delta(\omega)+$ regular terms. This is the signature for a solid with $f_{q}$ denoting its Debye-Waller factor. Hence, the strong coupling solution deals with a disordered solid; it is a model for an ideal glass state. If one increases the coupling constants smoothly from small to large values one finds a singular change of the solution from the ergodic liquid to the non-ergodic glass state, i.e. an idealized liquid-glass transition. For simple-liquid models the transition occurs upon cooling at some critical temperature $T_{c}$ or upon compression at some critical packing fraction $\varphi_{c}$ [20]. For the HSS model under study one finds $\varphi_{c} \approx 0.516$ [36]. If $\varphi$ increases above $\varphi^{c}$, the Debye-Waller factor $f_{q}$ increases above its value at the critical point, called the plateau $f_{q}^{c}$, as is demonstrated in Fig. 1. 
In the theory of crystalline solids one defines susceptibilities with respect to the restricted ensemble of a given arrested lattice. The MCT equations of motion allow for a similar formulation of the equations of motion for the glass state [22]. To see this, one has to map the density correlators $\phi_{q}$ to new ones $\hat{\phi}_{q}$ by

$$
\phi_{q}(t)=f_{q}+\left(1-f_{q}\right) \hat{\phi}_{q}(t)
$$

If one introduces new characteristic frequencies $\hat{\Omega}_{q}$ by

$$
\hat{\Omega}_{q}^{2}=\Omega_{q}^{2} /\left(1-f_{q}\right)
$$

one obtains the short time expansion $\hat{\phi}_{q}(t)=1-\frac{1}{2}\left(\hat{\Omega}_{q} t\right)^{2}+\cdots$ in analogy to what was found for $\phi_{q}(t)$. Substitution of these results into Eq. (1 motion with $\phi_{q}, \Omega_{q}$ and $m_{q}$ replaced by $\hat{\phi}_{q}, \hat{\Omega}_{q}$ and $\hat{m}_{q}$, respectively. Here the new relaxation kernels are related to the original ones by

$$
m_{q}(t)=C_{q}+\left(1+C_{q}\right) \hat{m}_{q}(t)
$$

For the dynamical susceptibility one obtains the formula $\chi_{q}(\omega)=\hat{\chi}_{q}\left[1+\omega \hat{\phi}_{q}(\omega)\right]$. The new correlator has a vanishing long time limit, $\hat{\phi}_{q}(t \rightarrow \infty)=0$, the Fourier-Laplace transform exhibits the property $\lim _{\omega \rightarrow 0} \omega \hat{\phi}_{q}(\omega)=0$. Since the equation of motion does not change its form, one gets as the analogue of Eq. (1B) the expression of the susceptibility $\chi_{q}(\omega)$ in terms of the polarization operator $\hat{m}_{q}(\omega)$ :

$$
\chi_{q}(\omega) / \hat{\chi}_{q}=-\hat{\Omega}_{q}^{2} /\left[\omega^{2}-\hat{\Omega}_{q}^{2}+\hat{\Omega}_{q}^{2} \omega \hat{m}_{q}(\omega)\right] \quad, \quad \hat{\chi}_{q}=\chi_{q}^{T}\left(1-f_{q}\right)
$$

Combining Eq. (3) with Eq. (4⿻) one concludes $\hat{m}_{q}(t \rightarrow \infty)=0$, i.e. the new kernel $\hat{m}_{q}(\omega)$ for the amorphous solid exhibits a regular zero-frequency behaviour: $\lim _{\omega \rightarrow 0} \omega \hat{m}_{q}(\omega)=0$.

The mentioned equation of motion for $\hat{\phi}_{q}(t)$ can be closed by combining Eqs. (4a) and (4c) with Eqs. (2a) and (20). One finds an expression of the new kernel as a new modecoupling functional $\hat{\mathcal{F}}$ of the new correlators:

$$
\hat{m}_{q}(t)=\hat{\mathcal{F}}_{q}[\hat{\phi}(t)]
$$


$\hat{\mathcal{F}}$ is a sum of a linear term $\hat{\mathcal{F}}^{(1)}$ and a quadratic one $\hat{\mathcal{F}}^{(2)}, \hat{\mathcal{F}}_{q}=\hat{\mathcal{F}}_{q}^{(1)}+\hat{\mathcal{F}}_{q}^{(2)}$, where:

$$
\begin{gathered}
\hat{\mathcal{F}}_{q}^{(1)}[\tilde{f}]=\sum_{k} \hat{V}_{q, k} \tilde{f}_{k} \quad, \quad \hat{V}_{q, k}=2\left(1-f_{q}\right) \sum_{p} V_{q, k p} f_{p}\left(1-f_{k}\right), \\
\hat{\mathcal{F}}_{q}^{(2)}[\tilde{f}]=\sum_{k p} \hat{V}_{q, k p} \tilde{f}_{k} \tilde{f}_{p} \quad, \quad \hat{V}_{q, k p}=\left(1-f_{q}\right) V_{q, k p}\left(1-f_{k}\right)\left(1-f_{p}\right) .
\end{gathered}
$$

As a result equations of motion are produced, which are of the same form as the MCT equations discussed in Sec.IIA. But in addition to the quadratic mode-coupling term there appears a linear one, and the values of the mode-coupling coefficients from Eq. (2b) are renormalized to the coefficients $\hat{V}_{q, k p}$.

The preceding Eqs. (5) are equivalent to equations relating the density-fluctuation spectra $\hat{\phi}_{q}^{\prime \prime}(\omega)$ with the kernel spectra $\hat{m}_{q}^{\prime \prime}(\omega)=\hat{m}_{q}^{(1) \prime \prime}(\omega)+\hat{m}_{q}^{(2) \prime \prime}(\omega)$ :

$$
\begin{gathered}
\hat{m}_{q}^{(1) \prime \prime}(\omega)=\sum_{k} \hat{V}_{q k} \hat{\phi}_{k}^{\prime \prime}(\omega) \\
\hat{m}_{q}^{(2) \prime \prime}(\omega)=\frac{1}{\pi} \sum_{k p} \int d \omega_{1} \int d \omega_{2} \hat{V}_{q, k p} \delta\left(\omega-\omega_{1}-\omega_{2}\right) \hat{\phi}_{k}^{\prime \prime}\left(\omega_{1}\right) \hat{\phi}_{p}^{\prime \prime}\left(\omega_{2}\right) .
\end{gathered}
$$

One can interprete Eqs. (4) and (5) using the language of the theory of boson fields, e.g. the phonon fields in crystals. $\hat{\chi}_{q}(\omega) / \hat{\chi}_{q}$ is the field propagator and $\hat{\Omega}_{q}$ is the bare-phonondispersion law. The kernel $\hat{m}_{q}(\omega)$ is the phonon self energy. Equations (5d) and (5d) are golden-rule expressions for the phonon-decay rates. Kernel $\hat{m}^{(1)}$ describes elastic scattering of the phonon from the disorder, produced by the amorphous glass structure. Kernel $\hat{m}^{(2)}$ deals with the decay of a phonon into two due to anharmonicities. The glass structure influences the decay rates via the Debye-Waller factors which enter Eqs. (5b) and (50) for $\hat{V}_{q, k}$ and $\hat{V}_{q, k p}$, respectively. The challenge is to evaluate this probability $\hat{m}_{q}^{\prime \prime}(\omega)$ self consistently; the decay depends on the same phonons as one wants to study.

\section{The glass-transition scenario}

Figure 2 exhibits the evolution of the dynamics with increase of the packing fraction $\varphi$. The wave vector $q=7.0$, used in the lower panel, is close to the structure-factor-peak 
position where the static susceptibility $\chi_{q}^{T} \propto S_{q}$ is high, while the wave vector $q=3.4$, used in the upper panel, deals with fluctuations where $\chi_{q}^{T}$ is very small (compare Fig. 1). Figure 3 shows the equivalent information for the fluctuation spectra $\phi_{q}^{\prime \prime}(\omega)$. In Ref. [40] a further set of diagrams for the wave vector $q=10.6$ can be found.

The curves for $\varphi<\varphi_{c}$ with label $n=1$ in Figs. 2 and 3 refer to the packing fraction $\varphi=0.276$. The correlators exhibit strongly-damped oscillations; and the ideal phonon resonances, to be expected for $m_{q}=0$, are altered to broadened bumps in the spectra. If the packing fraction increases into the interval $0.9 \varphi_{c} \leq \varphi<\varphi_{c}$, the oscillatory features almost disappear for $t>0.2$; and the shown spectra $\phi_{q}^{\prime \prime}(\omega)$ decrease monotonously with increasing $\omega$. Simultaneously, the decay to equilibrium is delayed to larger times. At the critical point the correlators approach the plateau $f_{q}^{c}$ in a stretched manner, as shown by the curves with label c in Fig. 2. This process, which is called critical decay, leads to a strong increase of the fluctuation spectra with decreasing frequency, as shown in Fig. 3. Increasing $\varphi$ above the critical packing fraction $\varphi_{c}$ the values for the long time limits $f_{q}$ increase. Since these limits are approached exponentially fast for $\varphi \neq \varphi_{c}$ [38], the correlation spectra become $\omega$-independent for low frequencies. The value for this white noise spectrum increases if $\varphi$ decreases towards $\varphi_{c}$; this is a precursor phenomenon of the glass melting at the transition point. If $\varphi$ is sufficiently far above $\varphi_{c}$, one observes oscillations again, as can be seen for the $\varphi=0.6$ result in the upper panel of Fig. 2. In this case, the correlation spectra are not anymore monotone functions of frequency. For $q=3.4$, there occur two peaks for $\omega>10$. The narrow peak is due to high-frequency phonon propagation. In addition there is an anomalous-oscillation peak (AOP) for $\omega \approx 80$. For $q=7$ a phonon peak is absent in the spectrum for $\varphi=0.6$ but an AOP is present, as is shown in the lower panel of Fig. 3.

The time scale for normal-state-liquid dynamics is set by the Debye frequency $\omega_{D}$. It is the same scale as for the dynamics of the crystalline state of matter. For the discussion of this normal condensed-matter dynamics it is sufficient to consider a window of, say, two decades around $2 \pi / \omega_{D}$. This is demonstrated in Fig. 8 for the liquid state with label $n=1$ or for the glass state with label $n=3$. If the time increases from 0.01 to 1 the correlators 
$\phi_{q}(t)$ decrease from 0.9 to the long time limit. All oscillations occur within this interval, which is also called the transient regime. The spectra for these states are located within a corresponding regime of microscopic excitations, extending roughly between 0.3 and 300 . For frequencies around and below 0.3 there is only white noise for the specified normal states. The glass transition is connected with a dynamics, called glassy dynamics, which occurs for times longer and frequencies smaller than the ones characterizing the transient. For reasons which can be understood by asymptotic expansions about the critical point [22], the dynamics is stretched over many decades. The glassy dynamics of the HSS is discussed comprehensively in Refs. [36,41]. It is impossible to view glassy dynamics adequately on linear scales for $t$ or $\omega$. Conventionally, one represents the results on logarithmic abscissas as done in Figs. 2 and 3. The bifurcation for $\varphi=\varphi_{c}$ or $T=T_{c}$ also modifies the transient dynamics. The subject of the paper is the study of these modifications.

\section{EVOLUTION OF THE TRANSIENT DYNAMICS}

\section{A. Anomalous-oscillation peaks and high-frequency phonons}

For a discussion of the transient motion it is sufficient to consider dynamical windows of about two orders of magnitude. These can be viewed more adequately on linear rather than on logarithmic abscissas. There is no reason to consider such fine tuning of control parameters relative to the critical point as is necessary for a study of structural relaxation. Therefore let us extract the relevant information for the following discussion from Figs. 2 and 3 and replot it as Figs. 4 and 5. Let us first consider the results for wave vector $q=7$. For the packing fraction $\varphi=0.60$ the particles are localized in such tight cages that the

square root $\delta r=\sqrt{\left\langle\delta r^{2}(t \rightarrow \infty)\right\rangle}$ of the long-time limit of the mean-squared displacement is only $5.0 \%$ of the particle diameter [41]. Thus one expects the particles to bounce in their cages with an average frequency of the order $\omega \approx 2 \pi v /(4 \cdot \delta r)=78$. This explains qualitatively the oscillation around the equilibrium value $f_{7.0}$ exhibited by $\phi_{7.0}(t)$ in Fig. \& 
for $t<0.3$ and the corresponding "peak" of the spectrum in Fig. 5. The maximum position $\omega_{P} \approx 75$ of this AOP is estimated well by the crude formula. The AOP differs qualitatively from the Lorentzian which one would expect for some damped harmonic oscillation. The low frequency part of the peak decreases more steeply with decreasing $\omega$ so that there appears some threshold near $\omega=40$. Below the threshold the spectrum is flatter than expected for the wing of a Lorentzian. If $\varphi$ decreases to 0.567 the cages widen so that $\delta r=0.070$ 41. This explains the increase of the oscillation period exhibited by curve $n=3$ in Fig. 4 and the corresponding downward shift of $\omega_{P}$ in Fig. 5. The shift is accompanied by a strong increase of the spectrum for $\omega \leq 10$. The integral of the inelastic spectrum also increases, reflecting the decrease of the elastic contribution $\pi f_{q}$ which is exhibited in Fig. 1. The described trends continue if $\varphi$ is decreased further to 0.540 (curves $n=4$ ). The threshold and the spectral minimum for small frequencies are now replaced by a central peak for $\omega \leq 20$. The maximum of the AOP, estimated from $\delta r=0.099$ [41] as $\omega_{P} \approx 40$, is buried under the tail of the central peak; it merely shows up as a shoulder. The correlator still exhibits some small oscillation before it reaches its long time limit $f_{7.0}=0.96$ for $t>0.2$, but it does not fall below $f_{7.0}$ anymore. At the critical point $\delta r=\delta r^{c}=0.183$ [41] and so one estimates a position $\omega_{P} \approx 21$. But the critical decay manifests itself by the appearance of a long time tail of $\phi_{7.0}(t)$. The approach to the asymptote $f_{7.0}^{c}=0.85$ cannot be demonstrated on the linear time axis used in Fig. 4. This tail leads to a strong enhancement of the central peak, so that the AOP cannot be identified. This trend continues if a packing fraction below the critical value is considered.

For the glass states the correlators $\phi_{3.4}(t)$ exhibit weakly damped oscillations which lead to nearly Lorentzian resonances for the spectra. These excitations are analogous to phonons in crystals. The softening of the glass with decreasing $\varphi$ leads to a decrease of the phonon frequency, but Fig. 5 demonstrates that for all $\varphi \geq \varphi_{c}$ the peak positions are located considerably above the maximum position $\omega_{P}$ of the AOP discussed in the preceding paragraph. One recognizes for the $\varphi=0.60$ result in Fig. At that the oscillations for $t \leq 0.1$ do not occur around the long time limit $f_{3.4}$. Rather the oscillation centre follows 
a curve discussed above for the bouncing in the cage. This is equivalent to the fact that the phonon resonance does not exhaust the spectrum $\phi_{3.4}^{\prime \prime}(\omega)$, rather it is placed on top of some background. The background exhibits a similar threshold as discussed above for the AOP of $\phi_{7.0}^{\prime \prime}(\omega)$, and with decreasing $\varphi$ it also follows the same pattern as described for the $q=7.0$ spectra. Apparently, the dynamics for $q=3.4$ illustrates a hybridization of the phonon dynamics with the modes building the AOP. The regular dependence of the MCT solutions on control-parameter variations are the reason why the results in Figs. 4 and 5 do not exhibit any drastic change if $\varphi$ is shifted from the glass state with label $n=4$, referring to $\epsilon=\left(\varphi-\varphi_{c}\right) / \varphi_{c}=0.0464$, through the critical point to the liquid state with $\epsilon=-0.0464$. But upon shifting the state into the liquid the phonon resonances get buried under the relaxation spectra.

The variation of the spectra with changes of the wave vector $q$ is demonstrated in Fig. 6 for the stiff-glass state with the packing fraction $\varphi=0.60$. For $q \leq 0.6$ a single peak of nearly Lorentzian shape exhausts the whole inelastic spectrum $\phi_{q}^{\prime \prime}(\omega)$. The resonance position follows the dispersion law of high-frequency sound $\Omega_{q}^{\max }=v_{\infty} q$. Here $v_{\infty}$ is the sound speed expected from the glass susceptibility $\hat{\chi}_{q=0}$, Eq. (4d]), $v_{\infty}=v_{0} / \sqrt{1-f_{q=0}}=75.8$. Also the half width of the resonance exhibits the quadratic wave-vector variation expected for sound in an elastic continuum: $\Gamma_{q}=\gamma \cdot q^{2}$. The single-peak shape of the spectra is found for all wave vectors up to about $q_{D} / 2$, where $q_{D}=(36 \pi \varphi)^{1 / 3}=4.08$ denotes the Debye wave vector of the system. Also the linear dispersion law continues up to these large $q$-values as is demonstrated in Fig. 77. However, for $q \geq 0.6$ the resonance width $\Gamma_{q}$ is somewhat larger than expected by extrapolating the asymptotic law $\Gamma_{q}=\gamma\left(\Omega_{q}^{\max } / v_{\infty}\right)^{2}$, as is shown in Fig. 8. The sound frequency $\Omega_{q}^{\max }$ reaches the position of the maximum of AOP at $q \approx 1.2$ and extends considerably beyond this value for larger $q$, as is shown for $q=1.8$ in Fig. 6.

If $\Omega_{q}^{\max }$ increases to the centre of the AOP the Ioffe-Regel limit is approached; i.e. the sound frequency becomes of the same order as the resonance width. In this case hybridization of high-frequency sound with the modes forming the AOP becomes important. The sound resonance is not Lorentzian anymore, as demonstrated in Fig. 6. For $q=1.4$ the threshold 
of the AOP near $\omega=40$ causes a shoulder on the low-frequency wing of the sound peak. With increasing $q$ a broad flat background spectrum is formed between the threshold and $\Omega_{q}^{\max }$ as shown in Fig. 6 for $q=1.8$ and 2.2. For wave vectors exceeding $q_{D} / 2$ the spectrum exhibits two maxima. The one at lower frequency is due to the AOP. The peak at higher frequencies is the continuation of the high-frequency sound resonance, which is increased because of a level repulsion effect, as demonstrated in Fig. 6 for $q$ between 2.2 and 5.4. The high-frequency-phonon frequency $\Omega_{q}^{\max }$ increases with $q$ increasing up to about $q_{D}$, and then it decreases again if $q$ increases up to about $1.5 q_{D}$. So the dispersion follows the pattern expected for a crystal phonon near the Brillouin zone boundary. The width $\Gamma_{q}$ of the high-frequency phonon for $2.5 \leq q \leq 5.0$ varies only weakly with changes of $q$. Also the AOP maximum $\omega_{P}$ does not change very much if $q$ increases from 2 to 5 , as is demonstrated by the open circles in Fig. 7. In a large wave-vector interval around the position of the first sharp diffraction peak of the structure factor $S_{q}$, the spectra do not exhibit a phonon peak but the AOP only. The spectral shape varies only weakly with changes of $q$, except near $q=8.7$; there a new splitting in two peaks occurs.

In Fig. 9 the spectroscopic parameters $\Omega_{q}^{\max }$ and $\Gamma_{q}$ of high-frequency sound are presented as function of the packing fraction. The resonance frequency decreases with decreasing $\varphi$, reflecting the softening of the glass state upon expansion. Remarkably, the damping $\Gamma_{q}$ does not vary much with changes of $\varphi$.

\section{B. A generalized-hydrodynamics description for the glass-state dynamics}

The description of hydrodynamic sound is obtained by coarse graining correlation functions so that fluctuations on microscopic scales for the space-and-time variations are averaged out. Coarse graining is equivalent to restricting functions like $\hat{\Omega}_{q}$ or $\hat{m}_{q}(\omega)$ to their leading order Taylor coefficient with respect to their $q$-and- $\omega$ dependence. In this manner one gets from Eq. (4d) the hydrodynamics description:

$$
\chi_{q}^{H}(\omega) / \hat{\chi}_{q}=\Omega_{q}^{H 2} /\left[\omega^{2}-\Omega_{q}^{H 2}+i \omega \Gamma_{q}^{H}\right]
$$


Here the dispersion law $\Omega_{q}^{H}$ and the damping $\Gamma_{q}^{H}$ are given by

$$
\Omega_{q}^{H}=v_{\infty} q \quad, \quad v_{\infty}^{2}=v_{0}^{2}(1+C) \quad, \Gamma_{q}^{H}=\gamma q^{2} \quad, \quad \gamma=v_{\infty}^{2} K^{\prime \prime}(\omega=0)
$$

where the notation $C=C_{q=0}$ and $K(t)=\hat{m}_{q=0}(t)$ is introduced. Because of Eqs. (2a), (3) and $(4 \mathrm{C})$ one can express $C$ and $K(t)$ in terms of the mode-coupling functional $\mathcal{F}_{0}=\mathcal{F}_{q=0}$ :

$$
C=\mathcal{F}_{0}[f], \quad K(t)=\left\{\mathcal{F}_{0}[\phi(t)]-C\right\} /(1+C)
$$

An explicit expression for the new functional $\mathcal{F}_{0}$ follows from Eqs. (2a) and (2b):

$$
\mathcal{F}_{0}[\tilde{f}]=\int_{0}^{\infty} V_{k} \tilde{f}_{k}^{2} d k
$$

where the weight factors $V_{k} \geq 0$ read

$$
V_{k}=\rho S_{q=0}\left[S_{k} k / 2 \pi\right]^{2}\left[c_{k}^{2}+\frac{2}{3}\left(k c_{k}^{\prime}\right) c_{k}+\frac{1}{5}\left(k c_{k}^{\prime}\right)^{2}\right]
$$

The integral in Eq. (8a) is to be understood as a Riemann sum over the wave-vector grid of $M$ terms as specified in Sec.IIA.

The hydrodynamics description is not suited to deal with high-frequency sound and the AOP. In order to identify the essence of these phenomena a generalized-hydrodynamics description shall be developed. It is obtained from Eq. (4d) via approximating kernel $\hat{m}_{q}(\omega)$ by its zero-wave-vector limit:

$$
\chi_{q}^{G H D}(\omega) / \hat{\chi}_{q}=\hat{\Omega}_{q}^{2} /\left[\omega^{2}-\hat{\Omega}_{q}^{2}(1-\omega K(\omega))\right]
$$

This formula extends the hydrodynamics approximation in two respects. Firstly and most importantly, the hydrodynamic damping constant $i \gamma / v_{\infty}^{2}$ is replaced by the frequencydependent function $K(\omega)=K^{\prime}(\omega)+i K^{\prime \prime}(\omega)$. Secondly, the full non-trivial $q$ dependence of $\hat{\Omega}_{q}$ is kept. According to Fig. 7 it is the $q$-dependence of $\hat{\Omega}_{q}$ which dominates that of the whole density-fluctuation spectra. If one intends to describe these spectra for wave vectors up to the inverse of the interparticle distances, one must not simplify $\hat{\Omega}_{q}$. However, if one is merely interested in the description of high-frequency sound so that $q$ is restricted 
to the regime below $q_{D} / 2$, say, one can replace $\hat{\Omega}_{q}$ in Eq. (9) by the hydrodynamics limit $\Omega_{q}^{H}=v_{\infty} q$. In this case, formula (9) has a form often used in acoustics, where $(1-\omega K(\omega))$ is called the dimensionless longitudinal elastic modulus. Let us also rewrite the mode-coupling formula (]) for $K(t)$ more transparently in analogy to Eqs. (5):

$$
\begin{gathered}
K(t)=K^{(1)}(t)+K^{(2)}(t) \\
K^{(1)}(t)=\int_{0}^{\infty} W_{k}^{(1)} \hat{\phi}_{k}(t) \quad, \quad W_{k}^{(1)}=2\left(1-f_{q=0}\right) V_{k} f_{k}\left(1-f_{k}\right), \\
K^{(2)}(t)=\int_{0}^{\infty} W_{k}^{(2)} \hat{\phi}_{k}(t)^{2} \quad, \quad W_{k}^{(2)}=\left(1-f_{q=0}\right) V_{k}\left(1-f_{k}\right)^{2} .
\end{gathered}
$$

In Fig. 6 representative MCT spectra for the glass states of the HSS are compared with the results of the generalized-hydrodynamics approximation. For the regime $q \leq 2$ the description by Eq. (9) with $\hat{\Omega}_{q}=v_{\infty} q$ is very good. This does not only hold for the treatment of the high-frequency-sound resonance but also for the background spectrum. But the generalized-hydrodynamics approximation yields reasonable results also for wave vectors extending up to and exceeding the value of the structure-factor-peak position $q_{\max } \approx 7$. In particular the subtle hybridization of the phonon with the modes underlying the AOP are treated semi quantitatively correctly. Hence, one concludes that Eqs. (9) and (10) separate the problem of the evolution of the transient dynamics into two parts. The first part concerns the dependence on the wave vectors. This is determined entirely by the quantities $\hat{\chi}_{q}$ and $\hat{\Omega}_{q}$. These functions are constructed from the structure factor $S_{q}$, whose dependence on $q$ and $\varphi$ is well understood. $S_{q}$ determines the characteristic frequency $\Omega_{q}$ and the thermodynamic susceptibility $\chi_{q}^{T}$. The MCT equations provide the glass form factors $f_{q}$, which are understood as well [20]. And the combination of this information yields $\hat{\Omega}_{q}$ and $\hat{\chi}_{q}$ via Eqs. (4b) and (4ad). Consequently, the remaining issue of this paper is to provide the understanding of the kernel $K(\omega)$.

Figure 10 exhibits the spectra $K^{\prime \prime}(\omega)$ and also the reactive parts of the modulus $\Delta(\omega)=$ $1-\omega K^{\prime}(\omega)$ for three representative glass states. The spectra $K^{\prime \prime}(\omega)$ are much simpler 
than those for the correlators since phonon resonances are absent. This property is the essential result achieved by the Zwanzig-Mori theory, and MCT preserves this property. The spectra of the kernels consist of some broad background and an AOP. The AOP of $K^{\prime \prime}(\omega)$ has quite a similar form as discussed in Sec.IIIA for $\phi_{7.0}^{\prime \prime}(\omega)$ and also its changes due to changes of $\varphi$ are similar. Formula (9) describes the hybridization of two "oscillators". One is a bare phonon with dispersion $\hat{\Omega}_{q}$ and the other is represented by the AOP spectrum. The hybridization problem is analogous to the one considered, for example, in the dynamical theory of light propagation in dielectric media. The bare phonon corresponds to the electromagnetic wave in vacuum and $1 /[1-\omega K(\omega)]$ is the analogue of the dielectric function. In lowest order one gets two resonances of the coupled system whose frequencies $\Omega_{q}^{\max }$ are obtained approximately as solution of the equation $\left(\Omega_{q}^{\max } / \hat{\Omega}_{q}\right)^{2}=\Delta\left(\hat{\Omega}_{q}^{\max }\right)$ and the damping is estimated by $\Gamma_{q}=\hat{\Omega}_{q}^{2} K^{\prime \prime}\left(\hat{\Omega}_{q}^{\max }\right)$. Elementary discussion of these equations with the aid of Fig. 10, which is left to the reader, explains qualitatively the findings reported in Figs. 6.9.

A digression might be useful concerning the different role played by the two equations for the susceptibility (110) and (4di). The crucial property of glassy dynamics is that kernel $m_{q}(\omega)$ in Eq.(1Bi) depends strongly on frequency $\omega$. In particular, this kernel has a large reactive part $m_{q}^{\prime}(\omega)$. Therefore it makes no sense to try a hydrodynamics approximation for Eq. (1D), based on $m_{q}(\omega) \approx m_{q}(\omega=0)=i m_{q}^{\prime \prime}(\omega=0)$. But let us consider a generalizedhydrodynamics approximation, defined by ignoring the wave vector dependence of kernel $m_{q}(\omega)$. Because of Eq. (2a) this limit is given by $\mathcal{F}_{0}[\phi(t)]$ and thus it can be expressed by $K(\omega)$ via Eq. (7):

$$
m_{q=0}(\omega)=[-C / \omega]+(1+C) K(\omega) .
$$

The kernel $m_{q=0}(\omega)$ exhibits the pole $[-C / \omega]$, which is the signature of the ideal glass state; other subtleties of the glassy dynamics are hidden in $K(\omega)$. Substitution of Eq. (11) for $m_{q}(\omega)$ in Eq. (1D) reproduces Eq. (9), except that the renormalized frequency $\hat{\Omega}_{q}$ is replaced by the approximation to Eq. (4b): $\Omega_{q} / \sqrt{1-f_{q=0}}$. For our model of the HSS structure there are no serious wave-vector dependencies of $f_{q}$ for $q \leq q_{D} / 2$. This means that high-frequency 
sound and the background spectrum for $q \leq 2$ can also be discussed on the basis of Eq. (11b). The dotted lines in Fig. 6 exhibit the corresponding results for $q=1.0$ and 3.4. However, Fig. 1 demonstrates that the relevant factor $1-f_{q}=1 /\left(1+C_{q}\right)$ varies by more than $100 \%$ for $q$ approaching and exceeding $q_{D}$. Therefore the generalized-hydrodynamics approximation based on Eq. (11) cannot be used to discuss, for example, $\phi_{7.0}^{\prime \prime}(\omega)$. The superiority of the generalized-hydrodynamics approximation based on Eq. (4d) rather than on Eqs. (13) and (11) results from the fact, that Eqs. (9) and (10) treat the glass structure as it comes out from Eq. (3), thus avoiding the additional small- $q$ approximation $C_{q} \approx C_{q=0}$.

\section{The stiff-glass approximation}

In this section Eqs. (11) and (2) shall be considered for large coupling coefficients $V_{q, k p}$. Let

us write symbolically $V_{q, k p}=O(1 / \eta)$, so that various quantities can be classified according to their power of the small parameter $\eta$. For the function $C_{q}$, which enters Eq. (3) for $f_{q}$, one gets large values: $C_{q}=O(1 / \eta)$. Thus $1-f_{q}=O(\eta)$. Stiff glass states are characterized by Debye-Waller factors close to unity, as is demonstrated by curve (a) in Fig. 11. Therefore one concludes from Eqs. (5b), (5c), (10b) and (10a) that the renormalized coupling coefficients decrease towards zero in the limit $\eta \rightarrow 0$ :

$$
\hat{V}_{q k}, W_{k}^{(1)}=O(\eta), \quad \hat{V}_{q, k p}, W_{k}^{(2)}=O\left(\eta^{2}\right)
$$

After eliminating the arrested glass structure as described in Sec.IIB, the remaining MCT equations (4d) and (5) deal with a weak-coupling situation if the system is in a deep glass state. Figure 11 demonstrates how the coefficients $W_{q}^{(1,2)}$ decrease with increasing packing fraction. Let us introduce for later use also the integrated coupling coefficients

$$
w_{1,2}=\int_{0}^{\infty} W_{k}^{(1,2)} d k
$$

For the three packing fractions $\varphi=0.600(0.567,0.540)$ dealt with in Fig. 11 one finds $w_{1}$ $=0.39(0.53,0.64)$ and $w_{2}=0.14(0.34,0.78)$ respectively. Let us recall from Sec.IIB that 
$\hat{V}_{q, k}$ and the derived quantities $W_{k}^{(1)}, w_{1}$ specify the interactions of density fluctuations with the arrested amorphous structure, while $\hat{V}_{q, k p}, W_{k}^{(2)}, w_{2}$ quantify two-mode-decay processes. One concludes furthermore that in the limit $\eta \rightarrow 0$ the two-mode contributions to the kernel $m_{q}(\omega)$ get suppressed relative to the one-mode contributions, in particular: $K^{(1)}(t)=$ $O(\eta), K^{(2)}(t)=O\left(\eta^{2}\right)$. This explains the result shown in Fig. 12: for the packing fraction $\varphi=0.60$ the one-mode contribution $K^{(1) \prime \prime}(\omega)$ provides the dominant part of the total spectrum $K^{\prime \prime}(\omega)$. The role played by the two contributions $K^{(1)}$ and $K^{(2)}$ is utterly different. The former yields the AOP and the latter provides a background spectrum.

To explain the AOP a stiff-glass approximation shall be analysed which is indicated by superscripts (1). It is defined by dropping the two-mode contribution to $\hat{m}_{q}(\omega)$ in Eq. (4d). Substituting the result into the formula $\phi_{q}^{(1)}(\omega)=\left[\chi_{q}^{(1)}(\omega) / \hat{\chi}_{q}-1\right] / \omega$ one gets

$$
\begin{gathered}
\phi_{q}^{(1)}(\omega)=-1 /\left[\omega-\hat{\Omega}_{q}^{2} /\left[\omega+i \nu_{q}+\hat{\Omega}_{q}^{2} m_{q}^{(1)}(\omega)\right]\right], \\
m_{q}^{(1)}(\omega)=\hat{\mathcal{F}}_{q}^{(1)}\left[\phi^{(1)}(\omega)\right] .
\end{gathered}
$$

In order to ease the discussion at the end of this section, a friction term proportional to $\nu_{q} \geq 0$ has been included in the formulas. It is equivalent to complementing $m_{q}^{(1) \prime \prime}(\omega)$ by a white noise spectrum. Unless emphasized otherwise, one may read the formulas with $\nu_{q}=0$. Equations (14) define a special model for the MCT and all general theorems quoted in Sec.IIA apply. If one would treat the mode-coupling coefficients $\hat{V}_{q k} \geq 0$ in the Eq. (5b) for the functional $\hat{\mathcal{F}}_{q}^{(1)}$ as free parameters, Eqs. (14) could exhibit glass transitions. For the discussion of such bifurcations the $M$ by $M$ matrix $C_{q k}=\left\{\partial \mathcal{F}_{q}[f] / \partial f_{k}\right\}\left(1-f_{k}\right)^{2}, q, k=$ $1,2, \ldots M$, which is called the stability matrix, plays an essential role. Glass transitions are characterized by the spectral radius $\mathrm{E}$ of matrix $\mathrm{C}$ to be unity, and for all other states one gets $E<1$ [22, 38]. One checks, that the stability matrix of the complete theory as defined by Eqs. (4) and (5) is the same as the one for the stiff-glass approximation, defined by Eqs. (14). Therefore Eqs. (14) with the coefficients $\hat{V}_{q k}$ defined in Eq. (5b) do not exhibit glass-transition points anymore; in particular $\phi_{q}^{(1)}(t \rightarrow \infty)=0$. Let us note also that matrix 
$\mathrm{C}$ is equivalent to matrix $\hat{V}: C_{q k}=\left(1-f_{q}\right) \hat{V}_{q k} /\left(1-f_{k}\right)$. Therefore also the spectral radius of $\hat{V}$ is $E$. Hence the resolvent $S=(1-\hat{V})^{-1}$ exists.

The $\omega=0$ limit of Eqs. (14) yields the linear equation $\sum_{k}\left(\delta_{q k}-\hat{V}_{q k}\right) m_{k}^{(1)}(\omega=0)=$ $i \sum_{k} \hat{V}_{q k} \nu_{k} / \hat{\Omega}_{k}^{2}$. For the relevant case $\nu_{k}=0$ one concludes that the zero-frequency spectrum of the kernel vanishes. Therefore

$$
m_{q}^{(1) \prime \prime}(\omega \rightarrow 0)=R_{q} \omega^{2}
$$

and this implies for the low frequency behavior of the correlator

$$
\phi_{q}^{(1) \prime \prime}(\omega \rightarrow 0)=(\pi / 2)\left[\delta\left(\omega-\Omega_{q}^{H}\right)+\delta\left(\omega+\Omega_{q}^{H}\right)\right]+R_{q} \omega^{2}
$$

Hence, one finds Rayleigh's law: the scattering probability $m_{q}^{(1) \prime \prime}(\omega)$ of low-frequency phonons from static density fluctuations varies proportional to $\omega^{2}$.

Let us simplify the stiff-glass approximation by embedding it into the generalizedhydrodynamics description. According to Sec.IIIB this amounts to approximating $m_{q}^{(1)}(\omega)$ by its $q=0$ value, denoted $K^{(1)}(\omega)$. Similarly one should write for the so far not specified friction coefficient $\nu_{q}=\tau \hat{\Omega}_{q}^{2}$. Let us denote the stiff-glass susceptibility resulting from Eq. (14a) within the generalized-hydrodynamics approximation by $\chi_{q}^{S G A}(\omega)=\hat{\chi}_{q}\left(\phi_{q}^{(1)}(\omega) \cdot \omega+1\right)$, so that

$$
\chi_{q}^{S G A}(\omega)=\hat{\chi}_{q} \hat{\Omega}_{q}^{2} /\left[\omega^{2}-\hat{\Omega}_{q}^{2}+\omega \hat{\Omega}_{q}^{2}\left[i \tau+K^{S G A}(\omega)\right]\right]
$$

The problem is reduced to evaluating the kernel $K^{S G A}(\omega)$ from the equation

$$
K^{S G A}(\omega)=-\int_{0}^{\infty} d k W_{k}^{(1)}\left\{1 /\left[\omega-\hat{\Omega}_{k}^{2} /\left[\omega+\hat{\Omega}_{k}^{2}\left(i \tau+K^{S G A}(\omega)\right]\right]\right\}\right.
$$

Again, the general theorems of MCT apply. However, since the mode coupling coefficients have been altered by substituting $\hat{V}_{0 k}$ for $\hat{V}_{q k}$, the stability matrix has been changed. Therefore it cannot be excluded that the spectral radius E reaches unity for the simplified theory - This would be an artifact of the generalized-hydrodynamics approximation and Eqs. (16) and (17) must not be used in such case. 
The generalized-hydrodynamics approximation simplifies the Eq. (15a) by the replacement of $R_{q}$ by its $q=0$ limit to be denoted by $R_{0}: K^{S G A \prime \prime}(\omega \rightarrow 0)=R_{0} \omega^{2}$. The coefficient $R_{0}$ is obtained by substituting Eq. (15b) into Eq. (10b). Using Eq. (8b) one gets

$$
R_{0}=\rho\left(S_{0} / v_{\infty}\right)^{3} c_{0}^{2} f_{0}\left(1-f_{0}\right)^{2} /\left[4 \pi^{2}\left(1-w_{1}\right)\right]
$$

The Rayleigh spectrum is included in Fig. 12. A huge magnification was necessary to make this contribution visible on the scale of $K^{\prime \prime}(\omega)$.

One infers from Fig. 0 that for $q>2$ the variations of the renormalized frequency $\hat{\Omega}_{q}$ with changes of the wave vector $q$ is suppressed relative to the ones of $\Omega_{q}$. One can get an estimation of the $k>q_{D}$ contribution to the integral (17) by replacing $\hat{\Omega}_{k}$ by some averaged value $\tilde{\Omega}$. Figure $\pi$ suggests $\tilde{\Omega}=120$ for $\varphi=0.60$. Introducing $\nu=\tau \tilde{\Omega}^{2}$ and indicating the results of the specified estimation by a tilde, Eq. (16) is equivalent to

$$
\phi_{q \geq q_{D}}^{S G A}(\omega) \approx \tilde{\phi}(\omega)=-1 /\left[\omega-\tilde{\Omega}^{2} /\left[\omega+i \nu+\tilde{\Omega}^{2} \tilde{K}(\omega)\right]\right]
$$

According to Fig. 11 the $k \leq q_{D}$ contributions to the integral in Eq. (17) can be neglected, and thus this equation for the kernel simplifies to

$$
K^{S G A}(\omega) \approx \tilde{K}(\omega)=w_{1} \tilde{\phi}(\omega)
$$

The solution of Eqs. (19) for $\tilde{K}$ and $\tilde{\phi}$ reads

$$
\begin{gathered}
\tilde{K}(\omega)=\left[\omega_{+} \omega_{-}-z(\omega)^{2}+\sqrt{z(\omega)^{2}-\omega_{-}^{2}} \cdot \sqrt{z(\omega)^{2}-\omega_{+}^{2}}\right] /(2 \omega), \\
z(\omega)^{2}=\omega(\omega+i \nu) / \tilde{\Omega}^{2}, \quad \omega_{ \pm}=1 \pm \sqrt{w_{1}} .
\end{gathered}
$$

The coupling constant $w_{1}$ takes over the role of the spectral radius of the stability matrix. In order avoid artifacts of the various approximations leading to the results (20), the formulas can be applied only for $w_{1} \leq 1$. Figure 13 demonstrates that Eqs. (20) with $\nu=0$ describe the AOP of $K^{\prime \prime}(\omega)$ for the HSS with $\varphi=0.60$ reasonably well. One could improve the description by trying better choices for $\tilde{\Omega}$, but this would not lead to any new insight. 
Figure 11 shows that also for the evaluation of the integral in Eq. (109) the contributions for $k<q_{D}$ can be ignored. A leading estimation for the two-mode kernel can thus be obtained as $\tilde{K}^{(2)}(t)=w_{2} \tilde{\phi}(t)^{2}$. Using Eq. (19b) one gets therefore

$$
\tilde{K}^{(2)}(\omega)=(1 / \pi)\left(w_{2} / w_{1}^{2}\right) \int \tilde{K}\left(\omega-\omega^{\prime}\right) \tilde{K}^{\prime \prime}\left(\omega^{\prime}\right) d \omega^{\prime}
$$

The zero-frequency limit leads to a trivial integral with the result $\tilde{K}^{(2) \prime \prime}(\omega=0)=\tau=$ $\left(w_{2} / \sqrt{w_{1}}\right) 8 /(3 \pi \tilde{\Omega})$. Going back to Eq. (10a) one concludes: the background term due to $K^{(2)}(t)$ could have been taken into account in its white noise approximation. This leads to the extension of the equation of motion by adding a friction term $\nu=\tilde{\Omega}^{2} \cdot \tau$. Such an extension is obtained by including this term in the formulas, as was done already in Eq. (14a) and the following formulas. The dotted line in Fig. 13 demonstrates that thereby all qualitative features of $K^{\prime \prime}(\omega)$ are understood. A further improvement is obtained by dropping the white noise approximation for the correction term $\tilde{K}^{(2)}$. This is done by replacing $\omega+i \nu$ by $\omega+\tilde{K}^{(2)}(\omega)$ in Eq. (20b). Figure 13 demonstrates that thereby a more satisfactory treatment of $K^{\prime \prime}(\omega)$ is obtained.

The periodic continued fraction for $\tilde{\phi}(\omega)$, which is defined by Eqs. (19), can be related to a Hilbert-Stieltjes integral: $\int_{-1}^{1} d x \sqrt{1-x^{2}} /(x-\zeta)=\pi(-\zeta+\sqrt{\zeta-1} \sqrt{\zeta+1})$. Thus one can express the normalized susceptibility $\tilde{\chi}(\omega)=[\omega \tilde{\phi}(\omega)+1]$ in the form:

$$
\begin{gathered}
\tilde{\chi}(\omega)=\int_{\omega_{-}^{2}}^{\omega_{+}^{2}} d \xi \tilde{\rho}(\xi) \chi_{\xi}(\omega), \\
\tilde{\rho}(\xi)=\sqrt{\left(\omega_{+}^{2}-\xi\right)\left(\xi-\omega_{-}^{2}\right)} /\left(2 \pi w_{1}\right), \\
\chi_{\xi}(\omega)=-\tilde{\Omega}^{2} /\left[\omega^{2}-\tilde{\Omega}^{2} \xi+i \omega \nu\right] .
\end{gathered}
$$

For the stiff-glass states the AOP is obtained as a superposition of undamped-harmonicoscillator spectra. The weight distribution $\tilde{\rho}(\xi)$ for the oscillators with frequency $\sqrt{\xi} \tilde{\Omega}$ extends from $\Omega_{-}=\omega_{-} \tilde{\Omega}$ to $\Omega_{+}=\omega_{+} \tilde{\Omega}$. If the approximate description is extended so that two-mode interactions are incorporated as white-noise-background spectrum for the 
fluctuating-force kernels, the results remain valid, but the oscillator dynamics has to include a Newtonian friction term, quantified by $\nu \geq 0$. A better description of the spectra for frequencies large compared to $\Omega_{-}$is obtained by acknowledging that the friction forces do not exhibit a white noise spectrum. This can be done by replacing $i \nu$ in Eq. (22c) by the kernel $\tilde{K}^{(2)}(\omega)$ from Eq. (21). The glass instability for $T \rightarrow T_{c}$ or $\varphi \rightarrow \varphi_{c}$ is connected with the approach of $w_{1}$ to unity, i.e., with the threshold $\Omega_{-}$approaching zero.

\section{SCHEMATIC-MODEL DISCUSSIONS}

\section{A. Models for anomalous-oscillation peaks}

The simplest MCT models deal with a single correlator, say $\phi(t)$. The Eq. (1a) remains valid with the subscript $q$ dropped. Generalizing Eq. (2a), the kernel $m(t)$ is written as mode-coupling function, specified by a series with coefficients $v_{n} \geq 0$ :

$$
m(t)=\mathcal{F}[\phi(t)]=\sum_{n=1}^{\infty} v_{n} \phi(t)^{n} .
$$

The long-time limit $f=\phi(t \rightarrow \infty)$ obeys Eq. (3): $f=\mathcal{F}[f] /(1+\mathcal{F}[f])$. For the glass states one can carry out the transformation discussed in Sec.IIB to get correlators $\hat{\phi}(t)$ and kernels $\hat{m}(t)$ with vanishing long time limits. Equations (4) and (5a) remain valid with the subscript

$q$ dropped. The new functional $\hat{\mathcal{F}}$ is again a power series. For the renormalized Taylor coefficients one gets in analogy to Eqs. (5b) and (5G): $\hat{v}_{n}=(1-f)^{n+1}\left[\partial^{n} \mathcal{F}[f] / \partial f^{n}\right] / n !, n=$ $1,2, \ldots$ The limit of a stiff glass is obtained if at least one of the coefficients $v_{n}$ becomes large. The number $\eta=1-f$ can be used as small parameter for the classification of terms. One finds $\hat{v}_{n}=O\left(\eta^{n}\right)$. Let us change the notation to $w_{1,2}=\hat{v}_{1,2}$ so that

$$
w_{1}=(1-f)^{2} \sum_{n=1}^{\infty} n v_{n} f^{n-1}, \quad w_{2}=(1-f)^{3} \sum_{n=2}^{\infty} n(n-1) v_{n} f^{n-2} .
$$

The stiff-glass approximation can be defined as in Sec.IIIC by approximating the kernel by the leading term $\hat{m}(t)=w_{1} \hat{\phi}(t)$. Let us change the notation to $\hat{\phi}=\tilde{\phi}$ and $\hat{\Omega}=\tilde{\Omega}$, so that

$$
\phi(t)=f+(1-f) \tilde{\phi}(t), \quad \tilde{\Omega}^{2}=\Omega^{2} /(1-f) .
$$


With $\tilde{K}(t)=\hat{m}(t)$ one arrives at the pair of equations (19).

Let us consider as an example the model specified by the functional $\mathcal{F}[f]=v_{1} f+v_{2} f^{2}$. It was introduced as the simplest one which can reproduce all possible anomalous exponents of the general MCT 22, 28]. Liquid-glass transitions occur on a line in the $v_{1}-v_{2}$ parameter plane. The line, where the long-time limit $f$ jumps from zero to $f^{c}=1-\lambda>0$, is a piece of a parabola with the representation $v_{1}^{c}=(2 \lambda-1) / \lambda^{2}, v_{2}^{c}=1 / \lambda^{2}, 0.5 \leq \lambda<1$. In Ref. [42] diagrams analogues to Figs. 2 and 3 can be found, which exhibit the evolution of glassy dynamics and the AOP upon shifting $\left(v_{1}, v_{2}\right)$ from the weak-coupling regime to the strong-coupling one. As path in the parameter plane a straight line was chosen: $v_{1,2}=$ $v_{1,2}^{c}(1+\epsilon), \lambda=0.7, \epsilon= \pm 1 / 4^{n}, n=0,1, \ldots$ The full lines in Fig. 14 reproduce three glass-state results. They are similar to what is demonstrated for the HSS in the lower panel of Fig. 5. The reason was explained in Sec.IIIC: these HSS spectra are described by the stiff-glass approximation and this approach yields the same formulas (19) as derived above for the schematic model. The dashed lines in Fig. 14 represent the leading order description by Eqs. (19) with $\nu=0$ and $w_{1}, \tilde{\Omega}$ determined by Eqs. (24) and (25). The dotted lines incorporate $\nu=\tilde{\Omega}^{2} \tilde{K}^{(2) \prime \prime}(\omega=0)$ evaluated from Eq. (21). The results for the $n=4$ spectra show that the extended stiff-glass approximation can describe the dynamics reasonably well for states which differ from the instability point by only $6.25 \%$. It was pointed out 42 that the schematic-model solutions in Fig. 14 fit qualitatively to the boson-peak scenario as reported for neutron-scattering data. It was shown 43] that the AOP of the specified model can be used to fit the boson-peak spectrum of orthoterphenyl as it was measured 44 by Raman scattering at $T=245 K=T_{c}-45 K$ for frequencies between $100 \mathrm{GHz}$ and 700 $\mathrm{GHz}$.

Usually, a one-component schematic model is too restrictive to deal quantitatively with experimental data. But one can construct more elaborate schematic models with the intention to mimic more features of the MCT. The perspective of such approach shall be indicated by results for the model used recently for the interpretation of scattering spectra of glassy 
liquids [29,31 34]. The model extends the one of the preceding paragraph by introducing a second correlator, to be denoted by $\phi^{s}(t)$. The equation of motion has the standard form of Eq. (1a) with $\Omega_{q}, \phi_{q}$, and $m_{q}$ replaced by $\Omega^{s}, \phi^{s}$, and $m^{s}$ respectively. The mode-coupling functional for $m^{s}$ is characterized by a single coupling constant $v_{s} \geq 0$ :

$$
m^{s}(t)=v_{s} \phi(t) \phi^{s}(t)
$$

The model was introduced for the description of tagged-particle motion in liquids 45.

The long-time limit $f^{s}=\phi^{s}(t \rightarrow \infty)$ is obtained from Eq. (3) as $f^{s}=1-1 /\left(f v_{s}\right)$. The transformation to a new correlator and a new kernel with vanishing long time limits can be done as explained in Sec.IIB: $\tilde{\Omega}^{s 2}=\Omega^{s 2} /\left(1-f^{s}\right) ; \hat{\phi}^{s}(t)=\left[\phi^{s}(t)-f^{s}\right] /\left(1-f^{s}\right)$. From Eq. (4c) one gets

$$
\begin{gathered}
\hat{m}^{s}(t)=K_{s}^{(1)}(t)+K_{s}^{(2)}(t), \\
K_{s}^{(1)}(t)=u \hat{\phi}(t)+w_{s} \hat{\phi}^{s}(t) \quad, \quad K_{s}^{(2)}(t)=\hat{v}_{s} \hat{\phi}(t) \hat{\phi}^{s}(t), \\
u=(1-f) f^{s} / f \quad, \quad w_{s}=\left(1-f^{s}\right) \quad, \quad \hat{v}_{s}=\left(1-f^{s}\right)(1-f) / f .
\end{gathered}
$$

The stiff-glass approximation for the two component model requires $(1-f)$ and $\left(1-f^{s}\right)$ to be small so that $K_{s}^{(2)}(t)$ can be neglected compared to $K_{s}^{(1)}(t)$. Let us denote the results by a tilde. The equations of motion, which specialize Eqs. (14), read:

$$
\begin{gathered}
\tilde{\phi}^{s}(\omega)=-1 /\left[\omega-\tilde{\Omega}^{s 2} /\left[\omega+\varphi(\omega)+\tilde{\Omega}_{s}^{2} \tilde{K}^{s}(\omega)\right]\right], \\
\tilde{K}^{s}(\omega)=w_{s} \tilde{\phi}^{s}(\omega) \quad, \quad \varphi(\omega)=\tilde{\Omega}^{s 2} u \tilde{\phi}(\omega) .
\end{gathered}
$$

This result is equivalent to Eqs. (19) except that the friction constant $i \nu$ is generalized to a friction function $\varphi(\omega)$. The solution can therefore be written in form of Eq. (20a) with $z(\omega)^{2}$ and $\omega_{ \pm}$replaced, respectively, by

$$
z^{s}(\omega)^{2}=\omega(\omega+\varphi(\omega)) / \tilde{\Omega}^{s 2}, \quad \omega_{ \pm}^{s}=1 \pm \sqrt{w_{s}} .
$$


In particular Eqs. (22) hold with the appropriate change in the notation. The susceptibility is a superposition with weight $\tilde{\rho}^{s}(\xi)$ of the functions

$$
\chi_{\xi}^{s}(\omega)=-\tilde{\Omega}^{s 2} /\left[\omega^{2}-\tilde{\Omega}^{s 2} \xi+\omega \varphi(\omega)\right] .
$$

This is a harmonic-oscillator susceptibility where the interaction with the background is included via a friction function $\varphi(\omega)$, dealing with the AOP of the surrounding.

Figure 15 shows solutions for three characteristic choices of $\Omega^{s}$. The results refer to the stiff glass-state discussed in Fig. 14 for the label $n=0$. The coupling $v^{s}=10 / f$ was chosen so that $1-f^{s}=0.10$. The stiff-glass approximation results are shown as dashed lines. For $\Omega^{s}=0.15 \Omega$ the resonance of the second correlator is located so far below the AOP of the first correlator, that there appears an AOP of $\phi^{s \prime}(\omega)$ quite similar as discussed for the one-component model. In this case $\varphi(\omega)$ in Eq. (30) only produces a renormalization of the frequencies $\tilde{\Omega}^{s} \sqrt{\xi}$ and a strongly suppressed background. For $\Omega^{s}=\Omega$ the resonances of $\chi_{\xi}^{s}$ are shifted upward because of level repulsion and the hybridization yields a broad background extending from the threshold of the AOP of the first correlator to the AOP position of the second one. For $\Omega^{s}=0.6 \Omega$ the spectrum $\phi^{s \prime \prime}(\omega)$ exhibits an AOP whose low frequency threshold and maximum position are close to the ones for the AOP of $\phi^{\prime \prime}(\omega)$. The hybridization causes a suppression of the high frequency spectrum. Therefore the AOP in $\phi^{s \prime \prime}(\omega)$ is more asymmetrical than the peak in $\phi^{\prime \prime}(\omega)$. The hybridization results have similarities to the ones discussed in connection with Fig. 6. Indeed, it was explained in Sec.IIIC that the phonon modes are influenced by the large-wave-vector modes which built the AOP, but that there is no feedback of the phonons for wave vector $q<q_{D}$ on the AOP. This is the same situation as treated by the specified schematic two-component model.

\section{B. Random-oscillator models}

To get more insight into Eqs. (22), the following problem shall be considered: evaluate the averaged dynamical susceptibility $\chi(\omega)$ of an ensemble of independent harmonic oscillators. The oscillators are specified by their mass $\mu$ and by their frequencies $\Omega(\xi)>0$. The 
latter depend on a random variable $\xi$. Its distribution shall be denoted by $\rho(\xi) ; \rho(\xi) \geq$ $0, \int \rho(\xi) d \xi=1$. It is no restriction of generality to assume $\Omega(\xi)^{2}=\alpha+\beta \xi, \beta>0$; but $\xi$ has to be restricted from below to insure stability. Let us choose as minimum for $\xi$ the value -1. Thus one can write $\Omega(\xi)^{2}=\Omega_{0}^{2}[1+w+2 \sqrt{w} \xi]$, so that $\Omega_{0}>0$ defines the frequency scale and $w, 0 \leq w<1$, characterizes the minimum frequency $\Omega_{-}=\Omega_{0}(1-\sqrt{w})$. Denoting averages by bars, $\overline{A(\xi)}=\int A(\xi) \rho(\xi) d \xi$, the quantity of interest is

$$
\chi(\omega)=\overline{-\mu /\left[\omega^{2}-\Omega(\xi)^{2}\right]} .
$$

Let us define a characteristic frequency $\Omega>0$ by $\Omega^{-2}=\overline{\Omega(\xi)^{-2}}$. It specifies the static susceptibility $\hat{\chi}=\mu / \Omega^{2}$. If brackets denote canonical averaging, defined with respect to the oscillator Hamiltonian $H_{\xi}=\left[\left(P^{2} / \mu\right)+\mu \Omega(\xi)^{2} Q^{2}\right] / 2$, one gets for the fluctuations of the momentum $\left\langle P^{2}\right\rangle=\mu^{2} v^{2}$ and of the displacement $\left\langle Q^{2}\right\rangle=v^{2} / \Omega(\xi)^{2}$, where $v$ denotes the thermal velocity. The time evolution of some variable $A=A(Q, P)$ can be written as usual in terms of a Liouvillian $\mathcal{L}: A(t)=\exp (i \mathcal{L} t) A$, where $i \mathcal{L} A=(\partial A / \partial Q) P / \mu-(\partial A / \partial P) \mu \Omega^{2}(\xi) Q$.

To embed the problem into the standard framework of correlation-function theory a scalar product shall be introduced in the space of variables $A, B \ldots$ by $(A \mid B)=\overline{\left\langle A^{*} B\right\rangle}$. The vectors $\mid Q)$ and $\mid P)$ are orthogonal and the normalization reads $(Q \mid Q)=v^{2} / \Omega^{2},(P \mid$ $P)=\mu^{2} v^{2}$. The Liouvillian is hermitian. The displacement correlator shall be defined by

$$
\phi(t)=(Q(t) \mid Q) /(Q \mid Q) .
$$

Its Fourier-Laplace transform can be written as Liouvillian-resolvent matrix element: $\phi(\omega)=\left(Q\left|[\mathcal{L}-\omega]^{-1}\right| Q\right) /(Q \mid Q)$. This quantity can now be represented within the Zwanzig-Mori formalism as a double fraction [35]: $\phi(\omega)=-1 /\left[\omega-\Omega^{2} /\left[\omega+\Omega^{2} m(\omega)\right]\right]$. The memory kernel $m(\omega)$ is the Fourier-Laplace transform of the fluctuating-force correlator

$$
m(t)=(F(t) \mid F) /\left(v^{2} \Omega^{2} \mu^{2}\right) .
$$

Here $F$ is the projection of the force $\partial_{t} P=-\mu \Omega^{2}(\xi) Q$ perpendicular to $\left.\mid Q\right)$ and $\mid P$ ):

$$
F=\mu\left[\Omega^{2}-\Omega^{2}(\xi)\right] Q .
$$


The time evolution in Eq. (33a) is generated by the reduced Liouvillian $\mathcal{L}^{\prime}, F(t)=$ $\exp \left(i \mathcal{L}^{\prime} t\right) F$, where $\mathcal{L}^{\prime}=\mathcal{P} \mathcal{L P}$ and $\mathcal{P}$ denoting the projector perpendicular to $\mid Q$ ) and $\mid P)$. The susceptibility is connected with the correlator as usual, $\chi(\omega) / \hat{\chi}=(\omega \phi(\omega)+1)$,

$$
\chi(\omega)=-\mu /\left[\omega^{2}-\Omega^{2}+\omega \Omega^{2} m(\omega)\right] .
$$

This exact representation of $\chi(\omega)$ in terms of kernel $m(\omega)$ is the analogue of Eq. (4d).

The essential point in the MCT is the approximation of the kernel $m(t)$ as modecoupling functional. The procedure [46], consists of two steps. Firstly, one reduces $F$ to the projection on the simplest modes contributing, and these are the pair modes $\mid Q \cdot \xi): F(t) \rightarrow \mid Q(t) \xi)(Q \xi \mid Q \xi)^{-1}(Q \xi \mid F)$. Secondly, one factorizes averages of products into products of averages: $(Q(t) \xi \mid Q \xi) \rightarrow(Q(t) \mid Q) \overline{\xi^{2}}$. As a result one finds

$$
m(t)=w_{1} \phi(t), \quad w_{1}={\overline{\xi\left[\left(\Omega^{2} / \Omega^{2}(\xi)\right)-1\right.}}^{2} / \overline{\xi^{2}} .
$$

These formulas are the analogue of Eqs. (41) and (5). They allow the approximate evaluation of $\chi(\omega)$ from the given input information $\Omega^{2}$ and $w_{1}$.

The Eqs. (33G) and (34) are equivalent to Eqs. (19) with $\tilde{\Omega}=\Omega=\hat{\Omega}$, and therefore the approximation for $\chi(\omega)$ can be written as noted in Eqs. (22). Thus, the presented MCT delivers an approximation for the oscillator susceptitiblity (31) in the sense that the general distribution $\rho(\xi)$ is approximated by $\tilde{\rho}(\xi)=2 \sqrt{1-\xi^{2}} / \pi$. If $\rho(\xi)=2 \sqrt{1-\xi^{2}} / \pi$ is chosen, one can check that $\tilde{\Omega}=\Omega_{0}$ and $w=w_{1}$. In this case MCT reproduces the exact result. Let us add, that the naive factorization for the kernel, $(F(t) \mid F) \simeq \mu^{2}(Q(t) \mid Q) \overline{\left[\Omega^{2}-\Omega(\xi)^{2}\right]^{2}}$, would not reproduce the exact result for the specified example; rather one would obtain Eq. (34) with an overestimated $w_{1}=w+w^{2}$.

\section{CONCLUSIONS}

Within mode-coupling theory (MCT) a critical temperature $T_{c}$ and a corresponding critical packing fraction $\varphi_{c}$ were introduced as the equilibrium-thermodynamics parameters 
characterizing the evolution of glassy dynamics. For silica, for example, $T_{c}$ is near $3300 \mathrm{~K}$ [47], and therefore all experiments quoted for this system in Sec.I deal with $T \ll T_{c}$ states. In this paper primarily states are studied where $T$ is so far below $T_{c}$ and $\varphi$ so far above $\varphi_{c}$ that structural-relaxation phenomena do not dominate the dynamics within the window of interest. These states are referred to as stiff-glass states. The dynamical window considered is the one of normal-condensed-matter physics, i.e. spectra are discussed within a twodecade regime for the frequency $\omega$ around and below the Debye frequency $\omega_{D}$. For the stiff-glass states the spectra of the $\alpha$-relaxation process are located at frequencies smaller than $\omega_{D} / 100$ and therefore it does not matter whether or not the quasielastic $\alpha$-peaks of the spectra are treated as elastic ones. Hence it is legitimate to use the basic version of the MCT which treats the crossover near $T_{c}$ as a sharp transition to an ideal glass at $T_{c}$. The derivation of the MCT formulas, in particular the one of Eq. (2b) for the modecoupling coefficients, is based on canonical-averaging properties. For temperatures below the calorimetric glass-transition temperature $T_{g}$, the system is in a quenched non-equilibrium state. From a rigorous point of view the application of MCT is therefore restricted to the regime $T>T_{g}$. However, experiments on high-frequency sound and on the boson peak do not indicate anomalies for $T$ near $T_{g}$. Thus it seems plausible that the results of the present paper can be used also for an interpretation of $T<T_{g}$ data.

A major finding of this paper is that there are "peaks" of the density-fluctuation spectra for wave vectors $q$ exceeding about half of the Debye-vector $q_{D}$, which are quite different from what one would expect for phonon resonances in liquids or crystals. These peaks, which we refer to as the anomalous-oscillation peaks (AOP), show the properties of the so-called boson-peak listed in Sec.I. First, as shown in Fig. 7, the position $\omega_{P}$ of the peak maximum is several times smaller than $\omega_{D}$. In this sense the AOP is due to soft modes. Second, according to Eqs. (22), the AOP is a superposition of harmonic-oscillator spectra, where different oscillators are specified by different frequencies. This can be shown since there is a well-defined strong-coupling limit of the MCT equations, referred to as stiff-glass limit, where the equations of motion simplify so much that all features of the AOP can be 
worked out by analytical calculations (Sec.IIIC). In this limit the continuous spectra are purely inhomogeneous ones. Third, there is a lower cutoff $\Omega_{-}$of the frequency distribution. This causes the low frequency wing of the AOP to decrease more steeply with decreasing $\omega$ than expected for a Lorentzian. The high frequency wing extends further out than the low frequency one so that the AOP is skewed. Fourth, as the packing fraction decreases also the frequencies $\omega_{P}$ and $\Omega_{-}$decrease, and simultaneously the intensity of the spectrum increases. This is shown in the lower panel of Fig. 5 and in Fig. 10. The critical point is characterized by the threshold $\Omega_{-}$approaching zero. In this sense one concludes, that the evolution of the AOP is related to the dynamical instability predicted by $\mathrm{MCT}$ for $\varphi=\varphi_{c}$ or $T=T_{c}$. In order to understand a further property of the AOP one has to acknowledge that the leading correction to the stiff-glass results introduces a damping for the oscillators. It is due to the decay of an oscillator mode into two modes caused by anharmonicities. A simplified treatment of this phenomenon only leads to a modification of the formulas by the introduction of a friction constant $\nu$ in Eq. (22). Thus the peak is superimposed on a flat background and the sharp thresholds are changed to some smooth but rapid crossover. Not much is modified in the center of the AOP spectrum provided $\nu$ is not too big. But with decreasing $\varphi$ or increasing $T$, the ratio $\nu / \Omega_{-}$becomes much larger than unity so that the oscillators of low frequency get overdamped. As a result one obtains the explanation of the fifth property, namely a central relaxation peak is formed if $\left(\varphi-\varphi_{c}\right) / \varphi_{c}$ is about $10 \%$. In this case the AOP merely appears as a shoulder of the quasielastic spectrum as shown by the $n=4$ curve in the lower panel of Fig. 5. Shifting the parameters even closer to the instability point, the AOP gets buried under the wing of the quasielastic peak. The elementary formulas (19) and (20) describe this feature of the MCT solutions reasonably well for $\varphi$ approaching $\varphi_{c}$ up to about $5 \%$. The cited results are quite general and are obtained even for the simplest schematic MCT models, as is demonstrated in Fig. 14. Sixth, in a further refinement of the description one acknowledges that the glass compressibility has a wave-vector dependence. This enters in the form of a characteristic frequency $\hat{\Omega}_{q}$, which can be considered as a bare phonon dispersion of the amourphous solid. It exhibits a maximum 
for $q$ near $q_{D}$ and a minimum near the structure-factor-peak position $q_{\text {max }}$ which in turn is near $2 q_{D}$. This $\hat{\Omega}_{q}$-versus $-q$ curve is similar to the one for the characteristic frequency $\Omega_{q}$ of the liquid which plays an essential role in the MCT equations (1a) and (1b). However, the oscillations of the Debye-Waller factor, shown in Fig. 1, imply via the renormalization formula (4b), that the ratio of the maximum to the minimum frequency is much smaller for $\hat{\Omega}_{q}$ than for $\Omega_{q}$. This can be inferred in detail by comparing the full line in Fig. 0 with the dashed one. Therefore, the maximum position of the AOP is only weakly $q$-dependent. Summarizing, we suggest that the MCT of the AOP provides the basis of a first-principle explanation of the so-called boson peak.

The formulated theory for the AOP has a transparent interpretation. MCT explains in the first place the formation of an effectively arrested density distribution. This amorphous structure is characterized by the same quantity used to characterize crystalline structures, viz. by the Debye-Waller factor $f_{q}$. Within the same formalism which leads to $f_{q}$, the equations of motion for the density fluctuations of this structure are obtained (Sec.IIB). Such unified treatment of the glass structure and its dynamics should be a feature of every microscopic theory since it is the same array of particles which forms the frozen structure and which carries the fluctuations. This unified treatment is especially important if one intends to study the dynamics near the instability limit of the structure. The equations of motion describe the decay of fluctuations into pairs and also the scattering of fluctuations from the arrested structure. For the stiff-glass states one finds the latter processes to overwhelm the former, as discussed in connection with Eq. (12). In the stiff-glass limit one finds that the particles are localized in their cages and harmonic oscillations of the particles with their cages are a good description of the relevant modes. In this extreme limit the total susceptibility is the one of a distribution of independent-oscillator responses, where the distribution of oscillator frequencies is caused by the distribution of sizes and shapes of the cages. MCT provides an approximation of the distribution of the frequency squares, Eq. (22b), and characterizes the drift of the distribution with changes of control parameters. It provides also results for the corrections to this limiting result, namely the appearance of 
homogeneous line broadening due to mode decay and coupling of the oscillations leading to weak wave-vector dependencies of the AOP parameters.

One achievement of MCT is the possibility to explain homogeneous line-broadening effects via a golden-rule mechanism as is suggested by Eqs. (5d) and (56). This aspect was used above in connection with the evaluation of the oscillator damping $\nu$ due to two-mode decay and also in connection with the derivation of Rayleigh's law, Eq. (15a). However, the interpretation of Eqs. (5d) and (5e) in the spirit of a golden rule is quite misleading, if the transition probabilities $\hat{V}$ are so large that the self-consistent solutions are qualitatively different from the ones obtained by a lowest-order approximation. In such cases the formulas can lead to an approximation theory for an inhomogeneous "line width" phenomenon. It was shown explicitly in Sec.IVB that MCT provides an approximation approach towards this phenomenon, and there is an example for which MCT reproduces the exact result for the inhomogeneous spectrum. The MCT for the AOP is based on the fact that this theory can handle homogeneous and inhomogeneous spectra within the same framework.

A side remark might be helpful. According to MCT all structural relaxation features are independent of the details of the microscopic equations of motion [23],40]. Therefore the existence of an $\mathrm{AOP}$ is of no relevance for understanding glassy dynamics or the glass transition. But the AOP provides an interesting piece of information on the arrested glass structure. The AOP is the result of a mapping of the cage distribution on the frequency axis. The mapping is done from the configuration space on the time axis via Newton's equations of motion and canonical averaging followed by a Fourier-cosine transform to get a spectrum.

The arrest of density fluctuations at the ideal liquid-glass transition is driven by the ones with a wave number $q$ near the structure-factor-peak position $q_{\max } \approx 2 q_{D}$, since for these wave numbers the liquid compressibility $\chi_{q}^{T} \propto S_{q}$ is large. The compressibility of simple dense liquids for $q<q_{D}$ is very small and therefore excitations with wave vectors from this domain, which corresponds to the first Brillouin zone of the crystalline phase, are not important for the evolution of structural relaxation and the glass transition. For the same reason one concludes that scattering processes of density fluctuations with $q<q_{D}$ are 
irrelevant for the formation of the AOP, as is demonstrated in Fig. 11. The soft complexes which cause the AOP are constructed from fluctuations with wave numbers near and above $q_{\text {max }}$. These conclusions are based on the MCT results for the Debye-Waller factors $f_{q}$. In order to produce the spectral peak in Fig. 12, $f_{q}$ has to be that large as shown by the uppermost curve in Fig. 11. This curve corresponds to $\epsilon=\left(\varphi-\varphi_{c}\right) / \varphi_{c}=0.16$. In Fig. B of Ref. 48 a measurement of $f_{q}$ for a hard-sphere colloid is documented for $3 \leq q d \leq 13$ and $\epsilon=0.11$. Since these experimental findings are close to the curve (a) in Fig. 1 we argue that the MCT results on the glass structure are in reasonable accord with the experimental facts. Let us emphasize, that the above reasoning refers to densely-packed systems of spherical particles. Obviously, in more complicated systems, like silica, the cages are not so tight like in a HSS. Therefore one can expect the soft configurations to be more subtle than discussed in this paper. The results in Sec.IVA for the two-component schematic model indicate, that the AOP can be more structured than obtained for the HSS. For systems with a low coordination number one can also expect intermediate range-order effects to play an important role. They enter, e.g., the coupling vertices in Eq. (2b) via the prepeaks of $S_{q}$ [16]. Whether MCT can handle the microscopic features leading to the AOP in complicated systems such as $\mathrm{ZnCl}_{2}$ is unclear at present. In particular, it is unclear whether MCT can contribute to understanding why the boson peak is more pronounced in strong glass formers like silica than in fragile glass formers like orthoterphenyl.

The preceding interpretation of the AOP suggests that these peaks appear in the spectra of all probing variables coupling to density fluctuations of short wave length. But different probing variables will weight the oscillating complexes differently and therefore the shape of the AOP and the position $\omega_{P}$ of the peak maximum will depend somewhat on the probe. Let us consider two examples. The first one deals with the tagged particle correlator $\phi_{q}^{s}(t)=$ $\left\langle\rho_{\vec{q}}^{s}(t) \rho_{\vec{q}}^{s *}\right\rangle$. Here $\rho_{\vec{q}}^{s}(t)=\exp (i \vec{q} \vec{r}(t))$ denotes the density fluctuation of a marked particle with position vector $\vec{r}(t)$. The spectrum $\phi_{q}^{s \prime \prime}(\omega)$ determines the incoherent neutron-scattering cross section. An exact equation for this quantity has the same form as Eq. (1a) with $\phi_{q}, m_{q}$ 
and $\Omega_{q}^{2}$ replaced by $\phi_{q}^{s}, m_{q}^{s}$ and $\Omega_{q}^{s 2}=v^{2} q^{2}$, respectively. The essential MCT equation is again the representation of the kernel as mode-coupling functional $m_{q}^{s}(t)=\sum_{k p} V_{q, k p}^{s} \phi_{k}(t) \phi_{p}^{s}(t)$. The coupling coefficients $V_{q, k p}^{s}$ are determined by the structure factor $S_{q}$. The mentioned equations have been derived and solved for the Lamb-Mössbauer factors $f_{q}^{s}=\phi_{q}^{s}(t \rightarrow \infty)$ of the HSS in Ref. [20]. Details of the discretization can be found in Ref. [41]. We have solved the cited equations for $\phi_{q}^{s}(t)$, and Fig. 16 exhibits fluctuation spectra for the packing fraction $\varphi=0.60$ for three wave vectors around the structure-factor-peak position. The shape of the peaks is only weakly $q$-dependent and the intensity varies nearly proportional to $q^{2}$. This finding is in agreement with neutron-scattering results and with moleculardynamics-simulation results for $\mathrm{ZnCl}_{2}$ [16] and silica [50].

The second example is related to the velocity correlator $\Psi(t)=\langle\dot{\vec{r}}(t) \dot{\vec{r}}\rangle /\left(3 v^{2}\right)$. For a harmonic system its spectrum determines the density of states $g(\omega)=2 \Psi^{\prime \prime}(\omega) / \pi$, normalized by $\int_{0}^{\infty} g(\omega) d \omega=1$. The velocity correlator can be extracted from $\phi_{q}^{s}$ [35. If one introduces the kernel $m^{(0)}(t)=\lim _{q \rightarrow 0} q^{2} m_{q}^{s}(t)$ one gets $\Psi(\omega)=-1 /\left[\omega+v^{2} m^{(0)}(\omega)\right]$. The localization length $r_{s}^{2}$, defined via the long time limit of the mean squared displacement $\left\langle\delta r^{2}(t \rightarrow \infty)\right\rangle=6 r_{s}^{2}$, determines the small- $q$ asymptote of $f_{q}^{s}$ and the pole of the kernel in the glass: $f_{q}^{s}=1-\left(q r_{s}\right)^{2}+O\left(q^{4}\right), m^{(0)}(\omega)=-1 /\left(\omega r_{s}^{2}\right)+O\left(\omega^{0}\right)$. One finds that the density of states vanishes proportional to $\omega^{2}$ for small frequencies as expected for an elastic continuum $g(\omega)=g_{0} \cdot \omega^{2}+O\left(\omega^{4}\right), g_{0}=m^{(0) \prime \prime}(\omega=0)\left(2 r_{s}^{4} / \pi v^{2}\right)$ [22]. Figure 17 exhibits a result for the HSS. The $\omega^{2}$-law is obtained only for frequencies below the threshold $\Omega_{-}$of the AOP. For larger frequencies the density of states is enhanced relative to the asymptotic law. The enhancement reaches a maximum of a factor of about 5 near the position of the AOP maximum, and it is about a factor 3 at the maximum of $g(\omega)$. For larger frequencies $g(\omega)$ gets suppressed as required by the normalization condition. The found enhancement phenomenon is in qualitative agreement with the experimental results reported for silica in Ref. [15] and with the simulation data in Ref. [13]. There is a consistency problem for the MCT. The prefactor in the $\omega^{2}$-law should be $g_{0}^{\prime}=\omega_{D}^{-3}\left[1+2\left(\omega_{D} / \omega_{D}^{\prime}\right)^{3}\right]$, where $\omega_{D}^{\prime}$ denotes the Debye-frequency for transversal sound. Since $g_{0}$ was calculated without any explicit 
reference to transversal sound, the approximations underlying MCT will lead to $g_{0} \neq g_{0}^{\prime}$. We did not study the solutions of the MCT equations for transversal excitations [20,22] in order to calculate $\omega_{D}^{\prime}$. Therefore we do not know the size of the error $g_{0}-g_{0}^{\prime}$. But if one estimates $\omega_{D}=\omega_{D}^{\prime}$ one gets $g_{0}^{\prime}=10.210^{-8}$, which is close to the value $g_{0}=9.210^{-8}$. This suggests that transversal excitations are taken into account to some extend implicitly in the formulas for $m_{q}^{s}(t)$.

It was already suggested earlier in connection with a discussion of soft-configuration models for glasses that the boson peak should be understood as result of quasi-harmonic oscillations of the system characterized by some distribution of oscillator potentials [51-54]. Obviously, the present theory is consistent with these phenomenological approaches. In Ref. 44] Raman spectra of glassy systems have been interpreted as a superposition of oscillator susceptibilities analogous to what is formulated in Eqs. (22). But there are two qualitative differences between this fit procedure and the present theory. In Ref. [44] the distribution $\tilde{\rho}(\xi)$ is taken as temperature independent, while Eq. (22b) for $\tilde{\rho}(\xi)$ describes the softening of the glass structure upon heating and, in particular, its instability for $T$ reaching $T_{c}$. In Ref. [44] the $T$ dependence of the spectra is introduced by replacing the damping constant $\nu$ by a Debye-function quantified by a temperature-dependent relaxation time. This viscoelastic theory leads to a Debye peak as quasi-elastic spectrum. Equations (22) do not lead to a quasi-elastic Debye spectrum as was explained in connection with Figs. 3 and 5. In Refs. [55,56 the effective-medium theory for percolation problems is modified to a theory for the displacement susceptibilities of a disordered harmonic lattice. For the susceptibility an expression similar to Eq. (16) is obtained where kernel $K^{S G A}(\omega)$ also describes the selfconsistent treatment of phonon scattering by the disorder. Even though the equation for $K^{S G A}(\omega)$ in Refs. [55,56] is quite different from Eq. (17), the solution looks similar to the dashed line in Fig. 13. However, the Rayleigh contribution in Ref. [56 is about $10^{6}$ times larger than the result based on Eq. (18). It was criticized [54 that in Refs. [55,56 the boson peak is constructed from fluctuations with wave vectors $q<q_{D}$ since thereby the role of long-wave-length fluctuations is overestimated. Hence the cited harmonic-lattice theory 
does not appear to be compatible with the theory studied in this paper.

Sound is obtained due to the interplay of inertia effects and stresses, which are built up due to compressions. The interplay is governed by the conservation laws for mass and momentum. The low-lying sound excitations interfere with other low-lying modes like structural relaxation and the oscillations building the AOP. Sound modes with wave vector $q \leq q_{D}$ and their interactions are not important for the explanations of structural relaxation [20]. In connection with Fig. 11 it was shown, that they are neither relevant for the explanation of the AOP. Therefore sound can be discussed within the standard procedure of acoustics by introducing a modulus. This procedure comes out within MCT as was explained in connection with Eq. (9), where $[1-\omega K(\omega)]$ is proportional to the complex modulus. Consequently, all results on high-frequency sound, discussed in this paper, are implications of the preceding results for $K(\omega)$. In this sense one concludes that high-frequency sound is a manifestation of the AOP. Let us contemplate the scales for frequency $\omega$ and wave number $q$ in order to be able to correlate the MCT results with some data. The boson-peak maximum observed for silica at $1 \mathrm{THz}[3]$ shall be compared with the maximum position $\omega_{P}=75$ for the $\varphi=0.60$ results for the AOP. The best resolution width $\Gamma_{\exp }$ achieved by the recent $\mathrm{X}$-ray-scattering experiments is $1.5 \mathrm{meV}$, i.e. in the units of this paper $\Gamma_{\exp } \approx 30$. The structure factor peak position $q_{\max }=1.5 \AA^{-1}$ for silica is to be compared with the value near 7 for the HSS. Hence the wave vector unit used is about $2 \mathrm{~nm}^{-1}$. Scattering experiments with the resolution $\Gamma_{\exp }$ have been done for silica for $q$ between $1 \mathrm{~nm}^{-1}$ and $4 \mathrm{~nm}^{-1}$ as can be inferred from Ref. [5] and the papers quoted there. X-ray-scattering experiments with larger $q$ are done with a resolution considerably worse than the cited $\Gamma_{\text {exp }}$. Thus the following discussion shall be restricted to wave vectors between $q=0.5$ and $q=2 \approx q_{D} / 2$. This wavevector interval corresponds to the interval for the sound frequency $\hat{\Omega}_{q}=v_{\infty} q$ between about 40 and 150 as is shown in Fig. 7 .

The first property of high-frequency sound follows from the upper panel of Fig. 10 . On the resolution scale $\Gamma_{\exp }$ the sound-dispersion law is $\Omega_{q}^{\max }=\hat{\Omega}_{q}=v_{\infty} q$. Here the sound speed $v_{\infty}$ is the one determined by the $q \rightarrow 0$ limit of the glass compressibility $\hat{\chi}_{q}$. 
The frequency-dependence of the reactive part of the modulus $1-\omega K^{\prime}(\omega)$ implies deviations from the strict linear law for $\Omega_{q}^{\max }$. According to Fig. 7 the deviations are predicted to occur on a $10 \%$ level. Thus they should be measurable if the resolution $\Gamma_{\exp }$ could be reduced by, say, a factor 5. Here some reservation has to be made. MCT does not contribute to the understanding of the structure factor $S_{q}$, rather it takes this quantity from other theories. Errors in $S_{q}$ will cause errors in the MCT results. It is notoriously difficult to calculate the small-q behavior of $S_{q}$. The Verlet-Weiss theory yields different results for $S_{q}$ than the used Percus-Yevick theory; and this causes also a small- $q$ behavior of $f_{q}$ which differs from the one exhibited in Fig. 11 [57]. Therefore the Verlet-Weiss theory will also lead to different $\hat{\Omega}_{q}$ which might change the results for $\Omega_{q}^{\max }$. It is not known how reliably the Percus-Yevick or the Verlet-Weiss theory describe the structure for packing fractions as large as 0.60.

If one smears out the spectrum $K^{\prime \prime}(\omega)$ shown in Fig. 10 with a resolution curve of width $\Gamma_{\text {exp }}$, one gets a result which is nearly $\omega$-independent within the dynamical window of interest: $v_{\infty}^{2} K^{\prime \prime}(\omega)=\gamma$. This explains the second property of high-frequency sound reported for the $\mathrm{X}$-ray-scattering results of silica [4, 5] and other systems [1, 7,8$]$ : the sound damping exhibits the hydrodynamic wave vector dependence $\Gamma_{q}=\gamma q^{2}$. Consequently, one can describe the whole measured spectrum by the damped-oscillator formulas (6a) and (6b) [1.2.5], albeit up to some background. The latter appears as white if viewed with resolution $\Gamma_{\exp }$. Naturally, it is difficult to separate this background from the one caused by other effects of the experimental setup. If one acknowledges the frequency dependence of $K(\omega)$, exhibited in Fig. 10, one concludes, that the formula $\Gamma_{q} \propto q^{2}$ is oversimplified. Figure 8 demonstrates the prediction, that a reduction of $\Gamma_{\exp }$ by a factor of three should be sufficient to detect an increase of $\Gamma_{q}$ above the $\Gamma_{q} \propto \Omega_{q}^{\max 2}$ asymptote if $q$ varies between 0.8 and 2 .

A crucial experimental finding is that the damping parameter $\Gamma_{q}$ does not vary much with changes of temperatures. This shows [4,7], that the sound damping mechanism cannot be due to anharmonicity-induced mode decay as known for phonons in crystals nor due to structural relaxation effects as studied in Brillouin-scattering spectroscopy of glassy liquids. The fast sound detected by neutron scattering [58] and molecular-dynamics simulation [59] in water, 
for example, occurs in a dynamical window where water exhibits its $\alpha$-relaxation process but no vibrations underlying a boson peak [60,61]. Therefore the fast-sound-damping in water depends appreciably on temperature and this dependence can be described reasonably within a visco-elastic model [59]. The insensitivity of the high-frequecy-sound damping on changes of control-parameters like temperature or density is indeed the third specification of the MCT results as shown in Fig. 9. In agreement with the assumptions of the phenomenological theories in Ref. 52,54 MCT explains the damping to be due to absorption of the sound mode by the oscillations building the AOP. The AOP depends on control parameters, as explained in connection with Figs. 10 and 14. Therefore $\Gamma_{q}$ is not strictly independent of control parameters. However, changes of density or temperature primarily redistribute the spectrum of $K^{\prime \prime}$ and thus the spectrum in the centre of the AOP does not change much. But, if the resolution $\Gamma_{\exp }$ could be reduced, a more subtle prediction could be tested. For $\Omega_{q}^{\max }$ near $140, \Gamma_{q}$ is $\varphi$-independent even if the system is driven as close to the critical point as shown by curve $\mathrm{n}=4$ in Fig. 10. For smaller $\Omega_{q}^{\max }$ the width $\Gamma_{q}$ increases with decreasing $\varphi$, and this is due to the appearance of the quasi-elastic relaxation peak of $K^{\prime \prime}(\omega)$. But for larger $\Omega_{q}^{\max }$, the damping constant should decrease with decreasing $\varphi$; in this case the softening of the system reduces the density of states for high-frequency-sound-decay processes. Let us emphasize that all MCT results discussed in this paper are solely based on the wave-vector and control-parameter dependence on the structure factor $S_{q}$. The explanation of the modulus and its drift with control-parameter changes is specified semi quantitatively by the three numbers only, which are specified in connection with Eqs. (20) and (21). Therefore our results are predicted to be valid for all systems with a structure factor similar to that of our HSS model, in particular for Lennard--Jones systems or van der Waals liquids like, e.g., orthoterphenyl.

The AOP of $K^{\prime \prime}(\omega)$ causes via Kramers-Kronig relations a frequency dependent reactive part $K^{\prime}(\omega)$. This implies that the sound resonance cannot exhaust the spectrum. Contrary to what holds for hydrodynamic sound, there must be a background spectrum. This is the fourth feature specified for high-frequency sound in Sec.I. Obviously, the detection of such 
background is difficult in view of many other reasons producing backgrounds for the experimental scattering signals. However, as explained in connection with the dashed lines in Figs. 13 and 14. MCT implies a fifth property: for $T \ll T_{c}$ or $\varphi \gg \varphi_{c}$, there is an effective lowfrequency threshold $\Omega_{-}$for the background. Such threshold can be used to discriminate the background due to density-fluctuation dynamics from the one due to experimental artifacts. There is a mathematically equivalent manner to formulate the physics of the background, which is better adopted to the present problem [10,17]. The dynamical structure factor $S(q, \omega)=S_{q} \phi_{q}^{\prime \prime}(\omega)$, considered as function of $q$ for fixed frequency $\omega$, represents the average of the square of the density-fluctuation Fourier components which oscillate with frequency $\omega$. The coherent contribution to these fluctuations leads to a peak at $q_{\omega}=\omega / v_{\infty}$. There is only a small contribution for $q<q_{\omega}$, since it is very difficult to excite long-wave-length fluctuations in densely packed systems. But there is a structureless background for $q>q_{\omega}$ extending to high values of $q$. It is caused by the large $-q$ density fluctuations produced by the distortions of the wave front due to the arrested amorphous glass structure. Figure 18 exhibits a MCT result for the HSS, which is in qualitative agreement with the simulation results reported in Ref. [10].

All specified MCT results for high-frequency sound and the AOP can be described well by the combination of the elementary formulas (9) and (20) with $K(\omega) \approx \tilde{K}(\omega)$. If regimes are considered, where relaxation can be ignored completely, one can use Eq. (20b) with $\nu=0$. In this case, only the two parameters $\tilde{\Omega}$ and $w_{1}$ need to be specified in order to quantify the result. Function $\tilde{K}(\omega)$ replaces the damping parameter $\gamma$ of the hydrodynamics-theory result, Eqs. (6). Introducing the third parameter $\nu$, the range of applicability of the results can be extended so, that structural-relaxation precursors are included. We suggest to use the cited formulas for an analysis of inelastic-X-ray-scattering data for high-frequency sound and of data for the evolution of the boson peak in glassy systems.

The derivation of the MCT equations is definitive and leads to a well-defined model for a non-linear dynamics. The point of view adopted in this paper is the following: results for the model are derived to provide explanations of previously unexplained features of the 
dynamics of liquids and glasses and to predict new results to be tested by future experiments. However, the "approximations" leading to the mode-coupling expression for the fluctuating-force kernel, Eqs. (2), are uncontrolled and therefore the range of validity of MCT is not understood. Let us conclude this paper by pointing out four open questions concerning the foundation of MCT, which are of particular relevance for the study of vibrational excitations. The first problem concerns the absence of any influence of transversal excitations on the dynamical structure factor. The results (15a) and (18) for Rayleigh's law account for the scattering of a longitudinal phonon into some other longitudinal wave, while the expected contribution due to conversion into transversal sound waves is missing. Similarly, the spectrum of the AOP is due to longitudinal excitations only, while Horbach et al. [18] report that transversal modes influence the boson-peak. The second problem concerns the mode-coupling approximation for the fluctuating-force kernel $m_{q}(t)$ for short times. For a Lennard-Jones system Eqs. (2a) and (2b) yield an overestimation of $m_{q}(t=0)$ compared to the values known from Monte-Carlo results. A procedure for eliminating this problem was suggested in Ref. [62], but it is unclear whether it can be used for supercooled systems. One also expects that the mode-coupling kernel should be complemented by some regular term [20]. In a simple treatment this would lead to an additional friction term $\nu_{q} \dot{\phi}_{q}(t)$ in Eq. (1a). For a dilute system $\nu_{q}$ could be calculated in the binary-collision approximation. The third unsolved problem is the evaluation of $\nu_{q}$ for the dense systems under consideration. Such friction term would imply an additive correction to the friction constant $\nu$ in Eqs. (20) and (22). If the resulting $\nu$ would be too big, the AOP could disappear in favour of an quasi-elastic spectrum due to overdamped oscillations. Whether this happens might depend on the structure of the system. As fourth unsettled question a cut-off problem has to be mentioned. For the large packing fraction $\varphi=0.60$, the results of our calculations would change somewhat if the used cutoff wave vector $q^{*} d=40$ was increased. This is due to the slow decrease towards zero of the direct correlation function $c_{q}$ of the HSS for $q$ tending to infinity. Since the introduction of a cutoff is equivalent to a softening of the hard-sphere potential it is conceivable that in the stiff-glass limit our model is rather a model for argon 
than for hard spheres. It would be desirable to examine whether this cutoff dependence disappears if a conventional regular interaction potential is used. A serious bottleneck for

such examination is the necessity to obtain reliable results for $c_{q}$ for strongly supercooled liquids.

\section{Acknowledgments}

We thank H.Z. Cummins, J. Horbach, A. Latz and W. Schirmacher for helpful discussions and critical comments on the manuscript. 


\section{REFERENCES}

[1] C. Masciovecchio, G. Ruocco, F. Sette, M. Krisch, R. Verbeni, U. Bergmann, and M. Soltwisch, Phys. Rev. Lett. 76, 3356 (1996).

[2] C. Masciovecchio, G. Monaco, G. Ruocco, et al., Phys. Rev. Lett. 80, 544 (1998).

[3] M. Foret, E. Courtens, R. Vacher, and J.-B. Suck, Phys. Rev. Lett. 77, 3831 (1996).

[4] P. Benassi, M. Krisch, C. Masciovecchio, V. Mazzacurati, G. Monaco, G. Ruocco, F. Sette, and R. Verbeni, Phys. Rev. Lett. 77, 3835 (1996).

[5] C. Masciovecchio, G. Ruocco, F. Sette, et al., Phys. Rev. B 55, 8049 (1997).

[6] E. Rat, M. Foret, E. Courtens, R. Vacher, M. Arai, and C. Masciovecchio, preprint (1999).

[7] G. Monaco, C. Masciovecchio, G. Ruocco, and F. Sette, Phys. Rev. Lett. 80, 2161 (1998).

[8] A. Matic, L. Börjesson, G. Ruocco, C. Masciovecchio, A. Mermet, F. Sette, and R. Verbeni, preprint (1999).

[9] V. Mazzacurati, G. Ruocco, and M. Sampoli, Europhys. Lett. 34, 681 (1996).

[10] M. Sampoli, P. Benassi, R. Dell'Anna, V. Mazzacurati, and G. Ruocco, Phil. Mag. B $77,473(1998)$.

[11] M. C. C. Ribeiro, M. Wilson, and P. A. Madden, J. Chem. Phys. 108, 9027 (1998).

[12] R. Dell'Anna, G. Ruocco, M. Sampoli, and G. Viliani, Phys. Rev. Lett. 80, 1236 (1998).

[13] S. N. Taraskin and S. R. Elliott, Phys. Rev. B (1999), submitted.

[14] N. J. Tao, G. Li, X. Chen, W. M. Du, and H. Z. Cummins, Phys. Rev. A 44, 6665 (1991). 
[15] U. Buchenau, M. Prager, N. Nücker, A. J. Dianoux, N. Ahmad, and W. A. Phillips, Phys. Rev. B 34, 5665 (1986).

[16] M. Foley, M. Wilson, and P. A. Madden, Phil. Mag. B 71, 557 (1995).

[17] S. N. Taraskin and S. R. Elliott, Phys. Rev. B 59, 8572 (1999).

[18] J. Horbach, W. Kob, and K. Binder, cond-mat/9901162 (unpublished).

[19] W. Schirmacher, G. Diezemann, and C. Ganter, Phys. Rev. Lett. 81, 136 (1998).

[20] U. Bengtzelius, W. Götze, and A. Sjölander, J. Phys. C 17, 5915 (1984).

[21] E. Leutheusser, Phys. Rev. A29, 2765 (1984).

[22] W. Götze, in Liquids, Freezing and Glass Transition, edited by J.-P. Hansen, D. Levesque, and J. Zinn-Justin (North-Holland, Amsterdam, 1991), p. 287.

[23] W. Götze and L. Sjögren, Rep. Prog. Phys. 55, 241 (1992).

[24] W. van Megen and S. M. Underwood, Phys. Rev. E 49, 4206 (1994).

[25] M. Nauroth and W. Kob, Phys. Rev. E 55, 657 (1997).

[26] T. Gleim, W. Kob, and K. Binder, Phys. Rev. Lett. 81, 4404 (1998).

[27] W. Götze, J. Phys.: Condensed Matter 11, A1 (1999).

[28] W. Götze, Z. Phys. B 56, 139 (1984).

[29] T. Franosch, W. Götze, M. R. Mayr, and A. P. Singh, Phys. Rev. E 55, 3183 (1997).

[30] V. Krakoviack, C. Alba-Simionesco, and M. Krauzmann, J. Chem. Phys. 107, 3417 (1997).

[31] A. P. Singh, G. Li, W. Götze, M. Fuchs, T. Franosch, and H. Z. Cummins, J. Non-Cryst. Solids 235-237, 66 (1998).

[32] B. Rufflé, J. Etrillard, B. Toudic, C. Ecolivet, G. Coddens, J. P. Ambroise, E. Gueguen, 
and R. Marchand, Phys. Rev. B 56, 11546 (1997).

[33] B. Rufflé, S. Beaufils, B. Toudic, C. Ecolive, A. Le Sauze, and R. Marchand, J. NonCryst. Solids 235-237, 244 (1998).

[34] B. Rufflé, C. Ecolivet, and B. Toudic, Europhys. Lett. 45, 591 (1999).

[35] J.-P. Hansen and I. R. McDonald, Theory of Simple Liquids, 2nd ed. (Academic Press, London, 1986).

[36] T. Franosch, M. Fuchs, W. Götze, M. R. Mayr, and A. P. Singh, Phys. Rev. E 55, 7153 (1997).

[37] R. Haussmann, Z. Phys. B 79, 143 (1990).

[38] W. Götze and L. Sjögren, J. Math. Analysis and Appl. 195, 230 (1995).

[39] R. Kubo, J. Phys. Soc. Japan 12, 570 (1957).

[40] T. Franosch, W. Götze, M. R. Mayr, and A. P. Singh, J. Non-Cryst. Solids 235-237, 71 (1998).

[41] M. Fuchs, W. Götze, and M. R. Mayr, Phys. Rev. E 58, 3384 (1998).

[42] W. Götze and L. Sjögren, Chem. Phys. 212, 47 (1996).

[43] S. P. Das, Phys. Rev. E 59, 3870 (1999).

[44] A. P. Sokolov, A. Kisliuk, D. Quitmann, A. Kudlik, and E. Rössler, J. Non-Cryst. Solids 172-174, 138 (1994).

[45] L. Sjögren, Phys. Rev. A 33, 1254 (1986).

[46] K. Kawasaki, Ann. Phys. (N.Y.) 61, 1 (1970).

[47] J. Horbach and W. Kob, cond-mat/9901067 (unpublished).

[48] W. van Megen, Transp. Theory Stat. Phys. 24, 1017 (1995). 
[49] S. Cusack and W. Doster, Biophys J. 58, 243 (1990).

[50] J. Horbach, W. Kob, and K. Binder (unpublished).

[51] V. G. Karpov, M. I. Klinger, and F. N. Ignat'ev, Sov. Phys. JETP 57, 439 (1983).

[52] M. I. Klinger, Physics Reports 165, 275 (1988).

[53] Yu. M. Galperin, V. L. Gurevich, and D. A. Parshin, Phys. Rev. B 32, 6873 (1985).

[54] U. Buchenau, Yu. M. Galperin, V. L. Gurevich, D. A. Parshin, M. A. Ramos, and H. R. Schober, Phys. Rev. B 46, 2798 (1992).

[55] W. Schirmacher and M. Wagner, in Dynamics of Disordered Materials, edited by D. Richter, A. J. Dianoux, and W. Petry (Springer, Berlin, 1989), pp. 231-234.

[56] W. Schirmacher and M. Wagener, Phil. Mag. B 65, 607 (1992).

[57] M. Fuchs, W. Götze, S. Hildebrand, and A. Latz, Z. Phys. B 87, 43 (1992).

[58] J. Teixeira, M. C. Bellissent-Funel, S. H. Chen, and B. Dorner, Phys. Rev. Lett. 54, 2681 (1985).

[59] F. Sciortino and S. Sastry, J. Chem. Phys. 100, 3881 (1994).

[60] A. P. Sokolov, J. Hurst, and D. Quitmann, Phys. Rev. B 51, 12865 (1995).

[61] F. Sciortiono, L. Fabbian, S.-H. Chen, and P. Tartaglia, Phys. Rev. E 56, 5397 (1997).

[62] L. Sjögren, Phys. Rev. A 22, 2866 (1980). 


\section{FIGURES}

FIG. 1. Debye-Waller factor $f_{q}$ (crosses) and one tenth of the Percus-Yevick static structure factor $S_{q}$ (lines) of a hard-sphere system (HSS) at the packing fractions $\varphi=0.600$ (a), $\varphi=0.540$ (b), and the critical packing fraction $\varphi=0.516$ (c). The inverse of the particle diameter, $1 / d$, is chosen as unit for the wave vector $q$.

FIG. 2. Density correlation functions $\Phi_{q}(t)$ of a HSS as a function of time $t$ for the wave numbers $q=3.4$ and $q=7.0$. The curves refer to the packing fractions $\varphi=0.6$ and $\varphi=\varphi_{c}\left(1 \pm 10^{-n / 3}\right)$ with $n$ given in the figure. Here $\varphi_{c} \approx 0.516$ denotes the critical packing fraction. The curves with label $c$ are the solutions at the critical point, which approach the long-time limits $f_{3.4}^{c}=0.36$ and $f_{7.0}^{c}=0.85$. The units of length and time have been chosen here and in all the following figures so that the hard-sphere diameter $d=1$ and the thermal velocity $v=2.5$.

FIG. 3. Fluctuation spectra $\Phi_{q}^{\prime \prime}(\omega)$ of the correlation functions shown in Fig. 2.

FIG. 4. Some of the correlators from Fig. 2 on a linear time axis. The full lines refer to glass states $(n=3: \varphi=0.567 ; n=4: 0.540)$ with the arrows indicating the long-time limits $f_{q}=\lim _{t \rightarrow 0} \Phi_{q}(t)$. The dashed lines with label $c$ exhibit the critical decay $(\varphi=0.516)$ and the lowest dashed curves refer to the liquid state for $n=4(\varphi=0.549)$.

FIG. 5. Fluctuation spectra $\Phi_{q}^{\prime \prime}(\omega)$ of the correlators shown in Fig. 4 .

FIG. 6. Spectra $\Phi_{q}^{\prime \prime}(\omega)$ (solid lines) of a HSS at packing fraction $\varphi=0.60$ for some wave numbers $q$. The dashed lines show the generalized-hydrodynamics approximation. For $q=1.0$ and $q=3.4$ the dotted lines show the generalized-hydrodynamics approximation with $f_{q}$ replaced by $f_{q=0}$. 
FIG. 7. Frequency $\Omega_{q}=v q / \sqrt{S_{q}}$ (dashed line), renormalized frequency $\hat{\Omega}_{q}=\Omega_{q} / \sqrt{1-f_{q}}$ (full line) and position of the global maximum $\Omega_{q}^{\max }$ of the spectrum (diamonds) of a HSS at packing fraction $\varphi=0.60$ as a function of the wave number $q$. For wave numbers at which two separate maxima of the spectrum can be identified the frequency position of the peak with the lower intensity is marked by the open circles. The vertical bars mark the frequency intervals where $\Phi_{q}^{\prime \prime}(\omega)$ exceeds half of the maximum intensity of the spectrum. The arrows point to the positions of the Debye wave number $q_{D}=(36 \pi \varphi)^{1 / 3}=4.08$ and the Debye frequency $\omega_{D}=v_{\infty} q_{D}=309$ corresponding to the high-frequency sound speed $v_{\infty}=75.8$. For $q=3.4(q=7.0)$ one gets $\Omega_{3.4}=77.0\left(\Omega_{7.0}=13.7\right)$ and $\hat{\Omega}_{3.4}=167\left(\hat{\Omega}_{7.0}=92.3\right)$.

FIG. 8. Width at half maximum $\Gamma_{q}$ (diamonds) of the high-frequency resonance of the spectrum $\Phi_{q}^{\prime \prime}(\omega)$ of the HSS for packing fraction $\varphi=0.60$ as a function of the resonance-maximum position $\Omega_{q}^{\max }$ for various wave vectors. The straight line represents the small-wave-vector asymptote $\Gamma_{q}=\gamma\left(\Omega_{q}^{\max } / v_{\infty}\right)^{2}=K^{\prime \prime}(\omega=0)\left(\Omega_{q}^{\max }\right)^{2}$ where $K^{\prime \prime}(\omega=0)=0.00182$.

FIG. 9. High-frequency sound-resonance position $\Omega_{q}^{\max }$ (diamonds) and resonance width $\Gamma_{q}$ (circles) as a function of the packing fraction, determined for $q=1.8$. The crosses exhibit the maximum position $\omega_{P}$ of the AOP of the density-fluctuation spectrum for wave vector $q=7.0$.

FIG. 10. Reactive parts of the moduli $\Delta(\omega)=1-\omega K^{\prime}(\omega)$ and spectra $K^{\prime \prime}(\omega)$ of the HSS for the packing fractions $\varphi=0.600$ (solid), $\varphi=0.567(n=3$, dashed) and $\varphi=0.540(n=4$, dotted).

FIG. 11. Mode-coupling coefficients $W_{q}^{(1)}$ and $W_{q}^{(2)}$ determining via Eqs. (10) the scattering and decay contributions, respectively, to the kernel $K$ of the HSS glass states. The curves with label $n=3$ and $n=4$ refer to the packing fractions $\varphi=0.567$ and $\varphi=0.540$, respectively. The insets show the coefficients for $q<3$ magnified by a factor 100 . 
FIG. 12. The spectrum $K^{\prime \prime}(\omega)$ of the kernel for the HSS at packing fraction $\varphi=0.60$ reproduced from Fig. 10 (full line). The dashed and the dotted curves exhibit the one-mode contribution $K^{(1) \prime \prime}(\omega)$ and the two-mode contribution $K^{(2) \prime \prime}(\omega)$, respectively. The full line with label $R$ denotes the Rayleigh contribution $R_{0} \omega^{2}\left(R_{0}=1.510^{-11}\right)$ magnified by a factor $10^{4}$.

FIG. 13. The full line reproduces the spectrum $K^{\prime \prime}(\omega)$ from Fig. 12. The dashed line exhibits the stiff-glass-approximation result $\tilde{K}^{\prime \prime}(\omega)$ from Eqs. (20) with $\tilde{\Omega}=120, w_{1}=0.388, \nu=0$. The dotted line is the extension of this approximation by incorporating $\nu=\tilde{\Omega}^{2} \tilde{K}^{(2) \prime \prime}(\omega=0) \approx 23$. The dot-dashed line shows the extension with $i \nu$ replaced by the kernel $\tilde{\Omega}^{2} \tilde{K}^{(2)}(\omega)$ from Eq. (21).

FIG. 14. Fluctuation spectra $\Phi^{\prime \prime}(\omega)$ for the one-component schematic model defined in Sec.IVA for a mode-coupling functional $\mathcal{F}[f]=v_{1} f+v_{2} f^{2}$ (solid lines) and $\Omega=1$. The states refer to the glass with distance parameters $\epsilon=1 / 4^{n}, n=0,1,2$ (compare text). The dashed line exhibit the stiff-glass approximation given by Eqs. (20) with $\nu=0$. The dotted lines show the extended description including a $\nu \neq 0$ which was evaluated from the $\omega=0$ limit of Eq. (21).

FIG. 15. Spectra $\Phi^{s \prime \prime}(\omega)$ of the solutions for the second correlator of the two-component model defined in Sec.IVA with $v^{s}$ chosen so that $f^{s}=0.9$. The first correlator needed as an input for the memory kernel (26) is the one corresponding to the $n=0$ curve in Fig. 14. The dashed lines are the stiff-glass approximations, Eqs. (28), to these curves. The arrows indicate, which axis corresponds to the curves.

FIG. 16. Rescaled tagged-particle-density-fluctuation spectra $10^{6} \Phi_{q}^{s \prime \prime}(\omega) / q^{2}$ of the HSS for the packing fraction $\varphi=0.600$ at wave numbers $q=3.4$ (solid), $q=7.0$ (dashed), and $q=10.6$ (dotted).

FIG. 17. Density of states $g(\omega)$ of a HSS, calculated for the packing fraction $\varphi=0.60$. The dashed line shows $g(\omega)=9.2 \times 10^{-8} \omega^{2}$, describing the density-of-states asymptote at small frequencies. The arrow marks the Debye frequency $\omega_{D}=309$ for the longitudinal sound. 
FIG. 18. Dynamic structure factor $S(q, \omega)=S_{q} \Phi_{q}^{\prime \prime}(\omega)$ of the HSS for packing fraction $\varphi=0.60$ as a function of the wave number $q$ at some fixed frequencies $\omega$. The Debye vector is $q_{D}=4.08$; threshold and maximum of the boson peak are located near $\omega=45$ and $\omega=85$ respectively (compare Fig. 12). The lines are guides to the eye. 


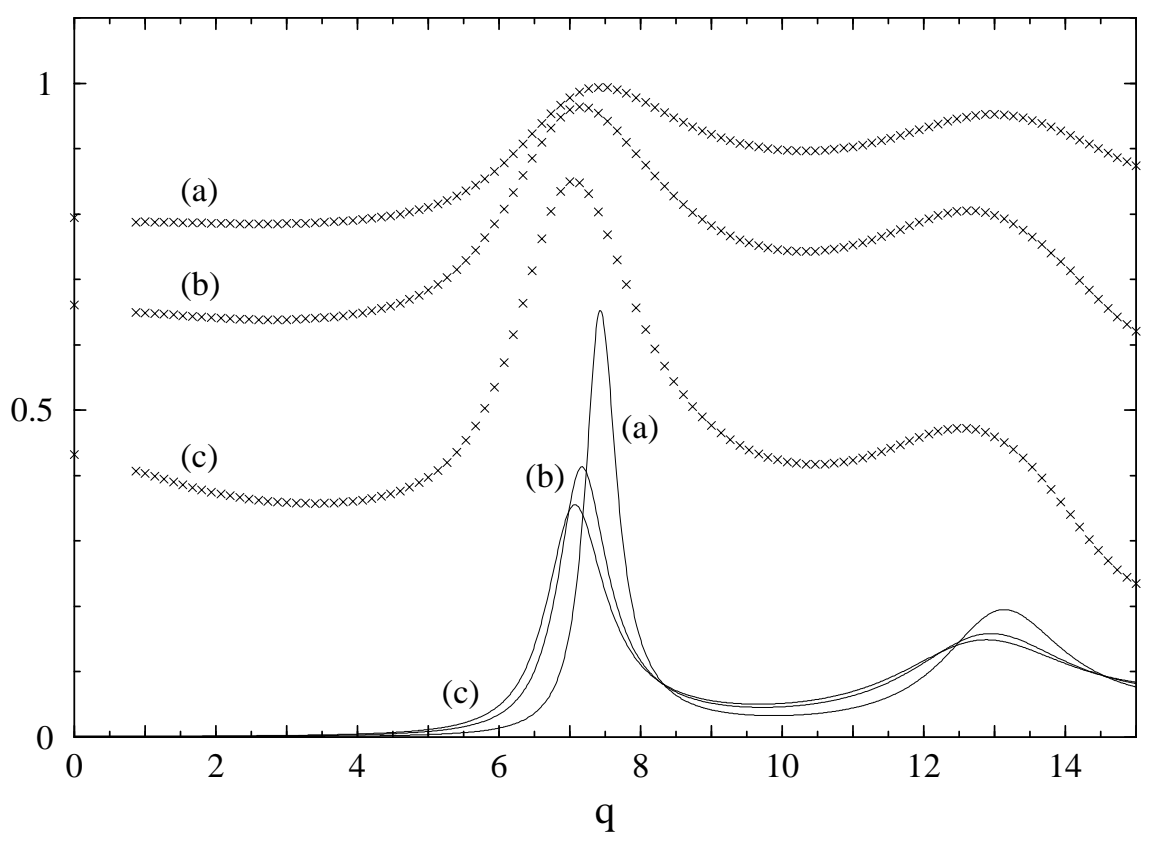

Figure 1: W. Götze and M. R. Mayr 


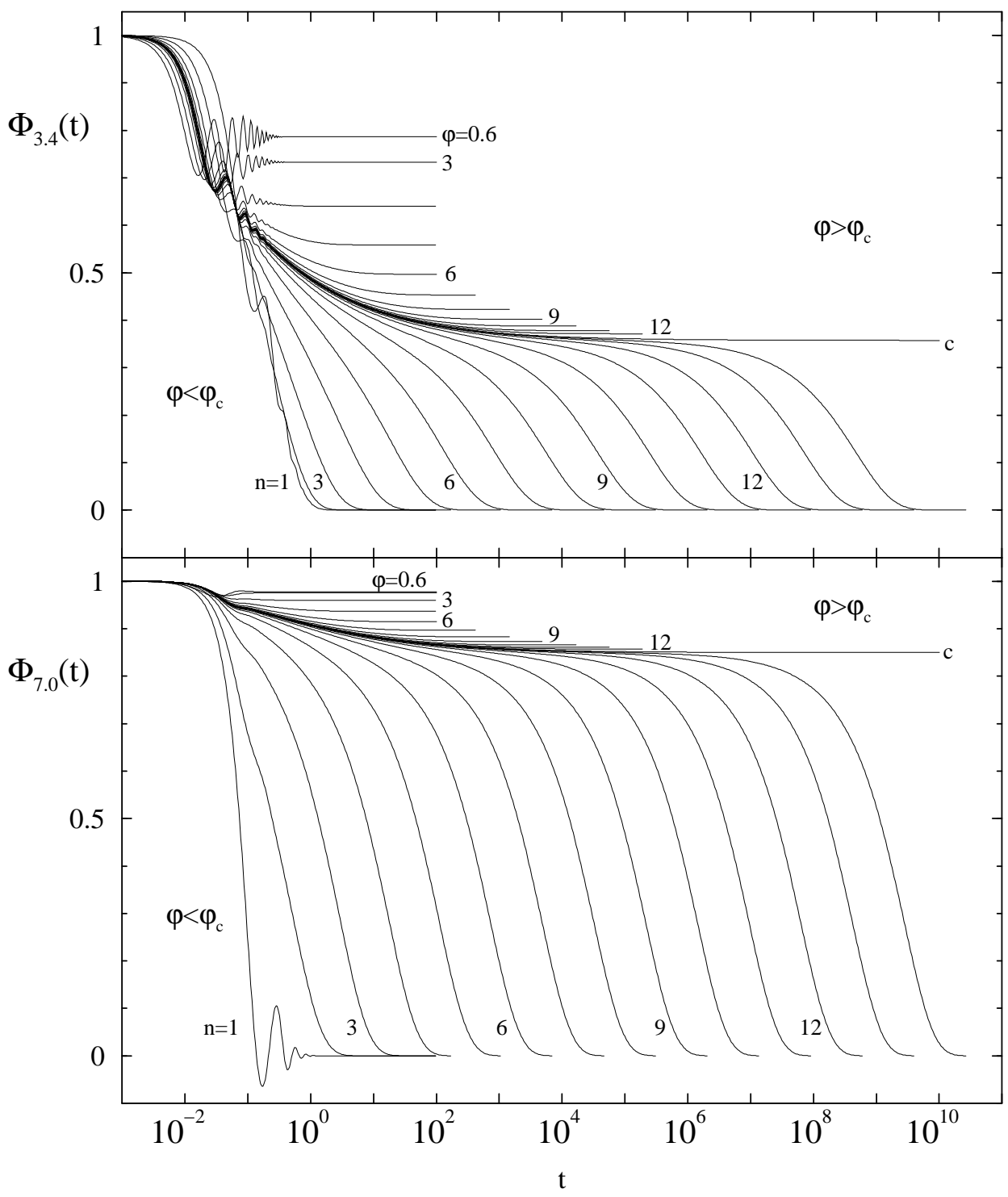

Figure 2: W. Götze and M. R. Mayr 


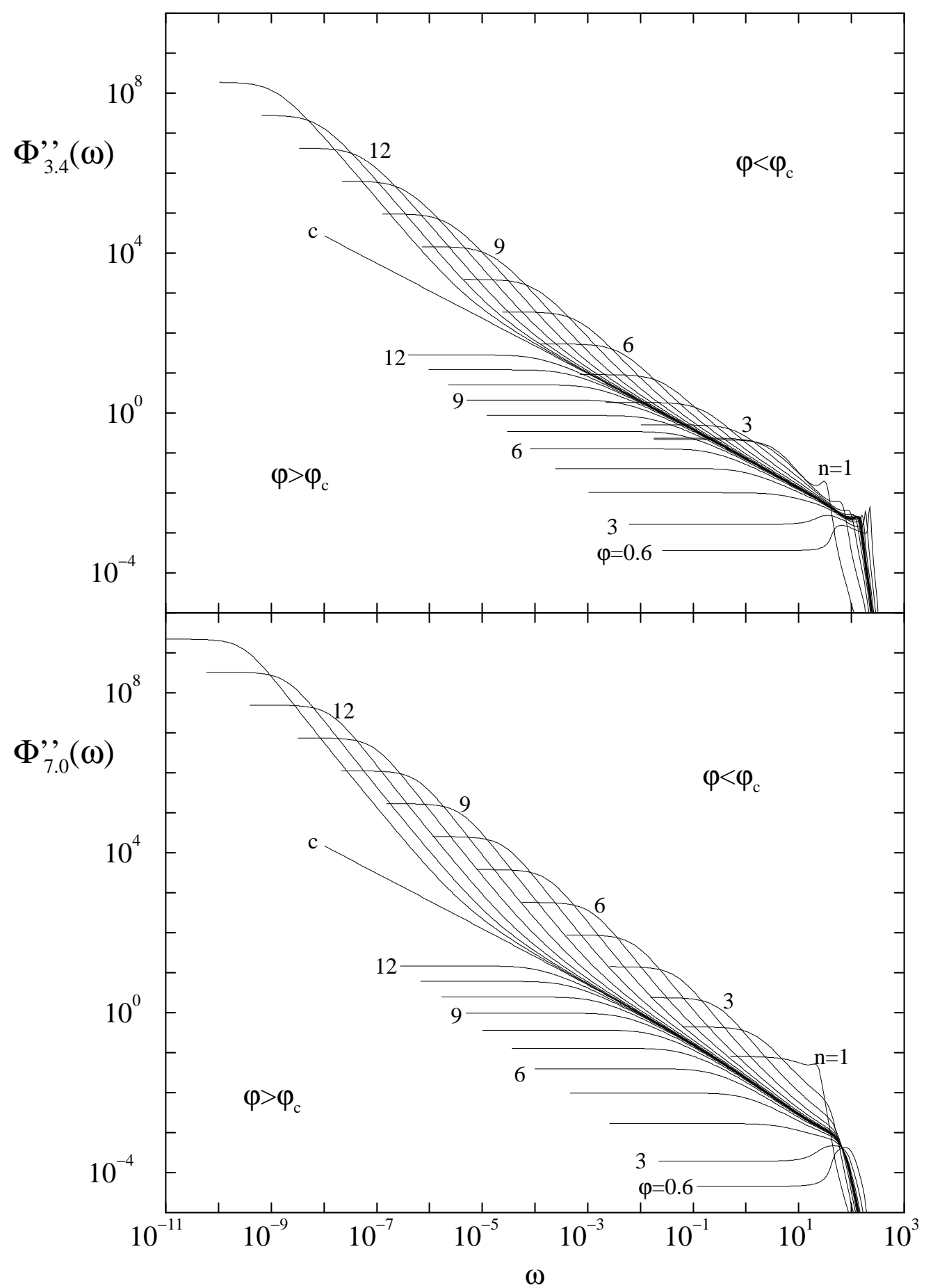

Figure 3: W. Götze and M. R. Mayr 


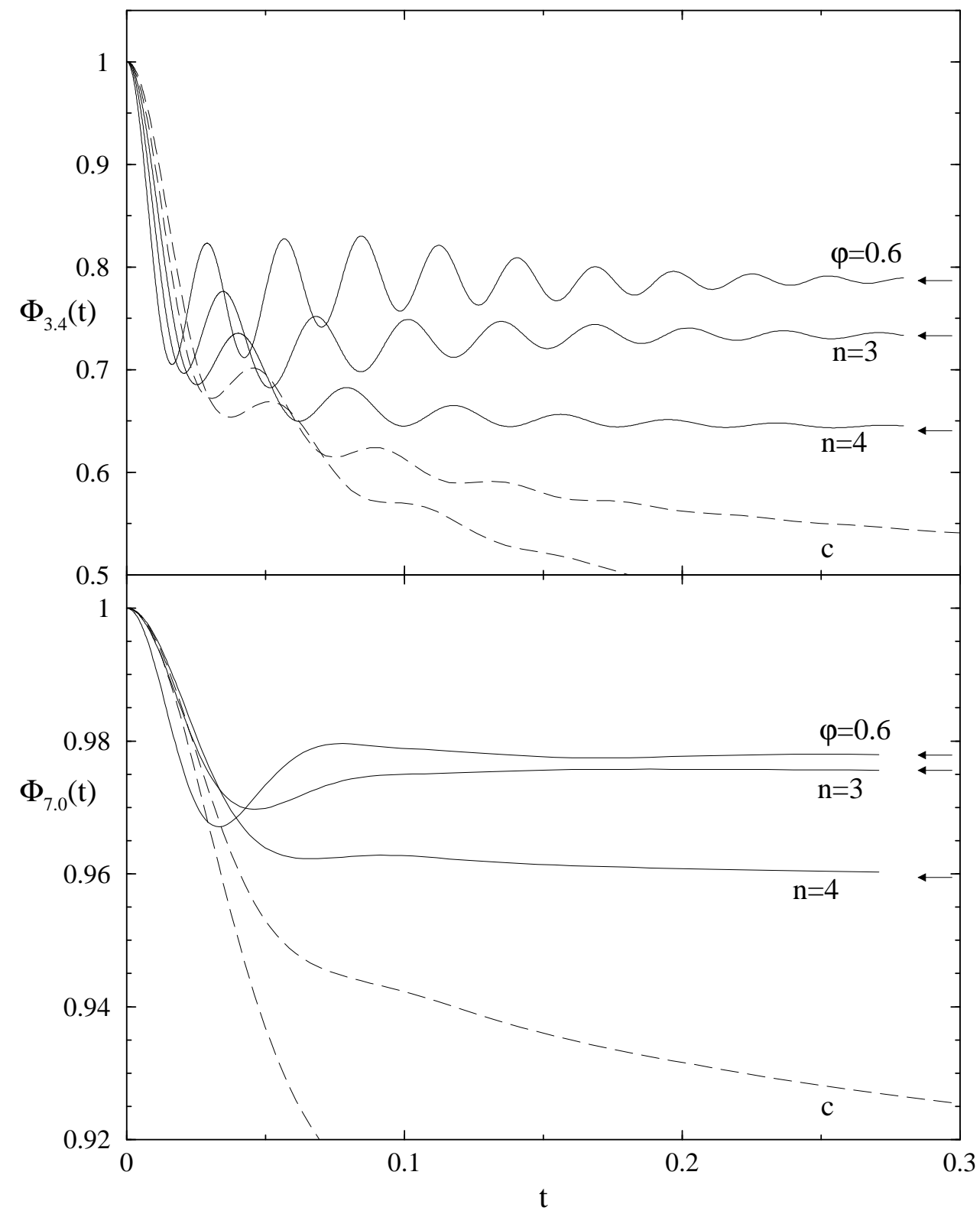

Figure 4: W. Götze and M. R. Mayr 


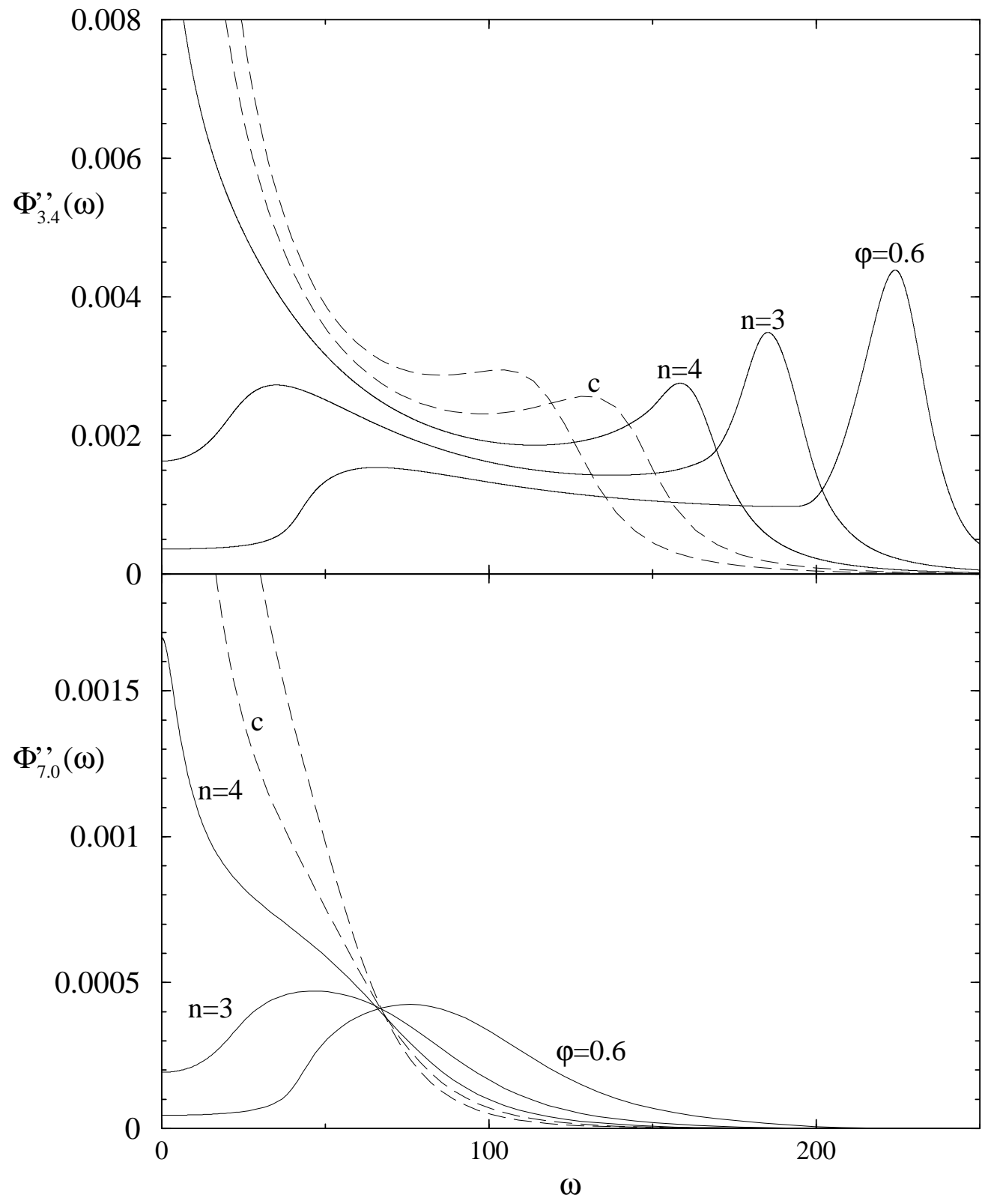

Figure 5: W. Götze and M. R. Mayr 


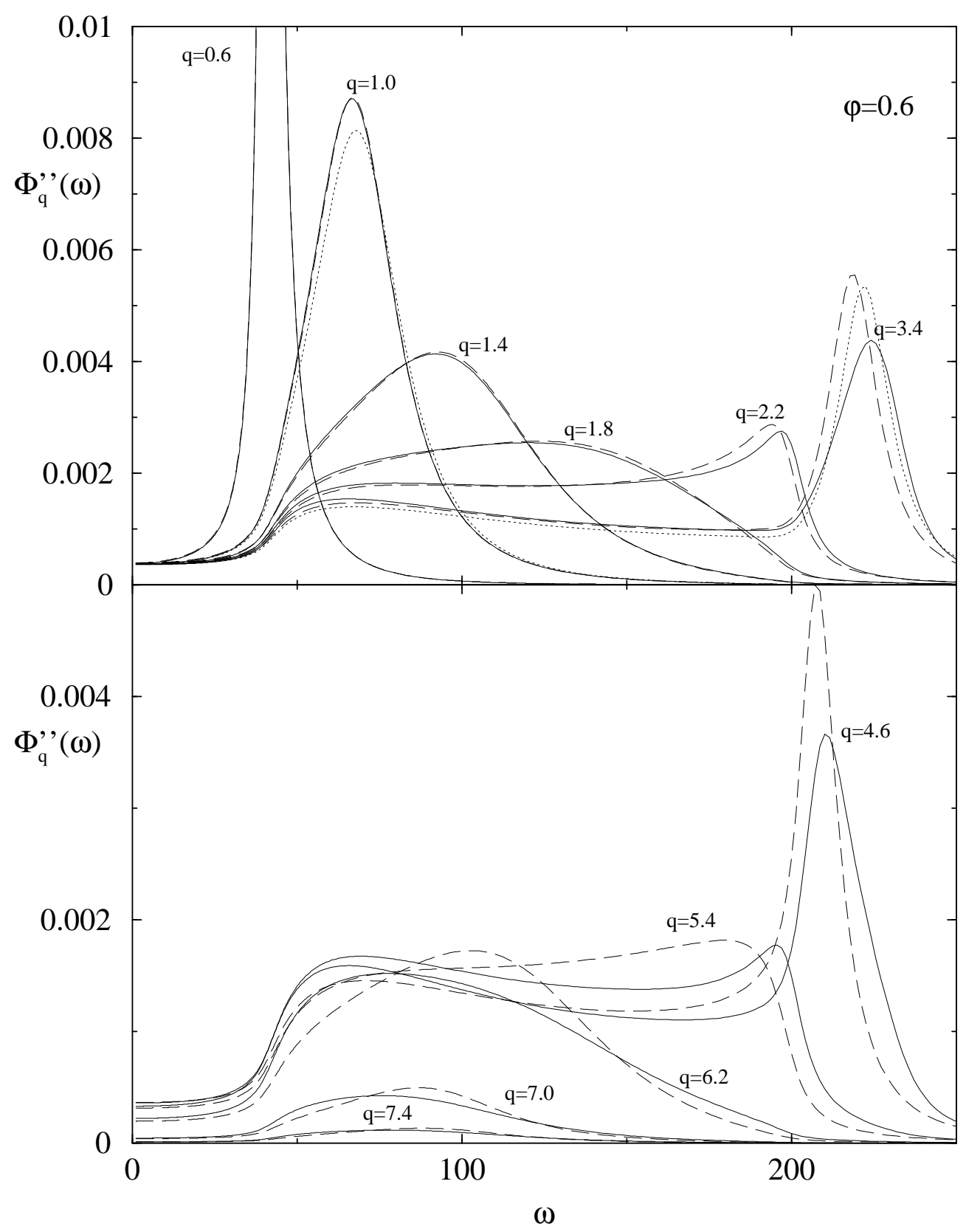

Figure 6: W. Götze and M. R. Mayr 


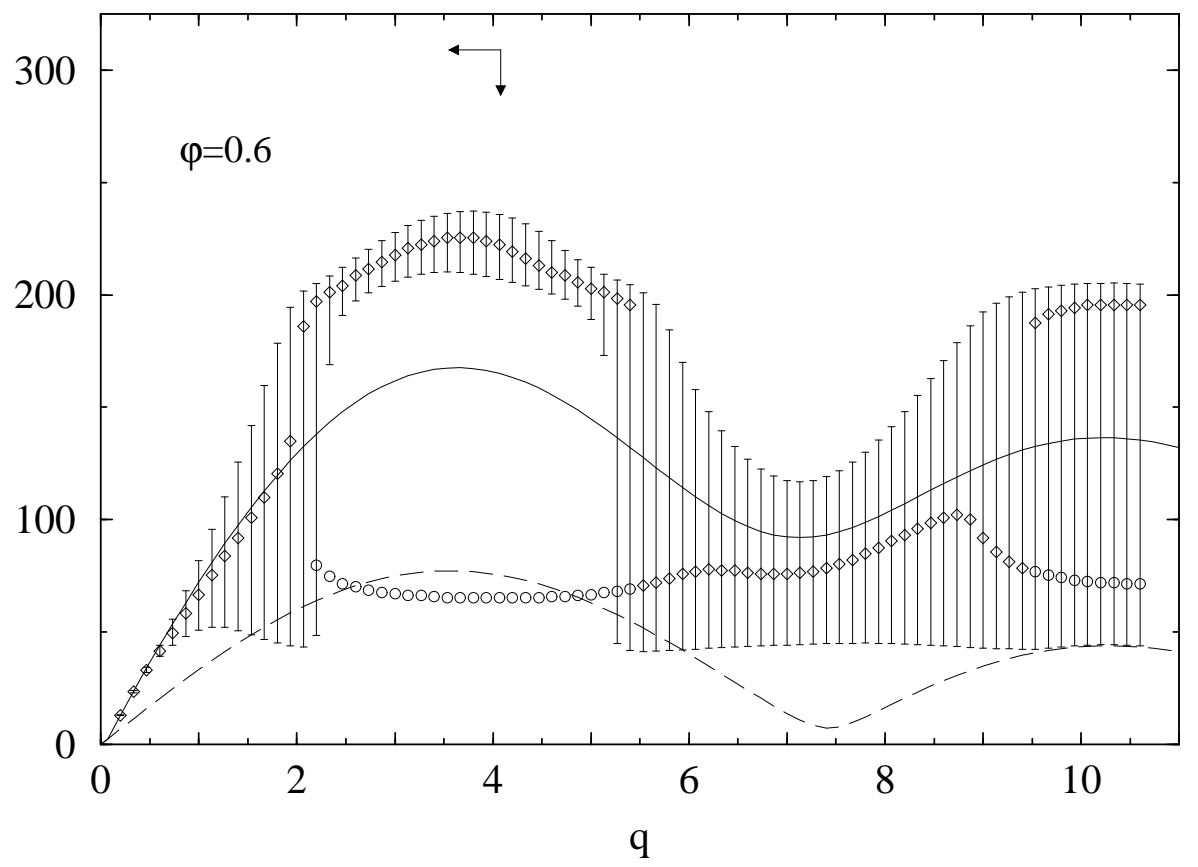

Figure 7: W. Götze and M. R. Mayr 


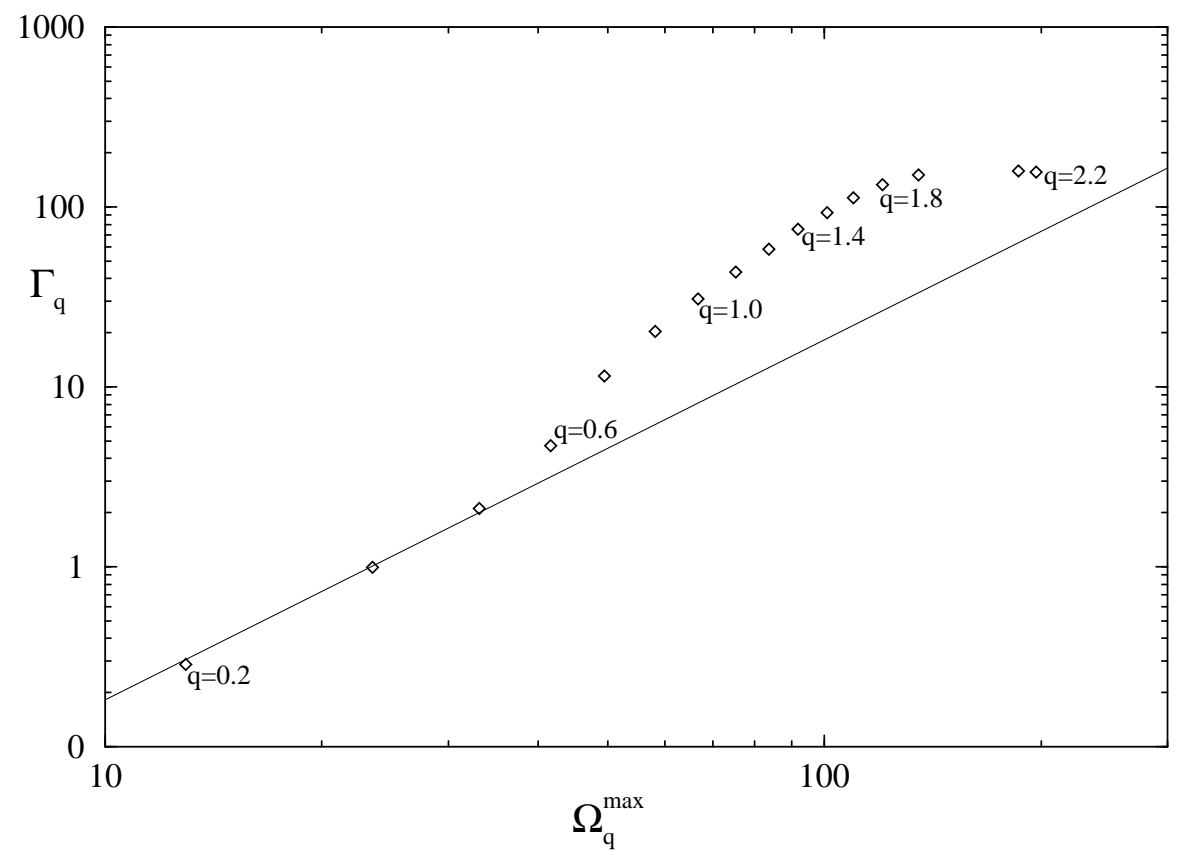

Figure 8: W. Götze and M. R. Mayr 


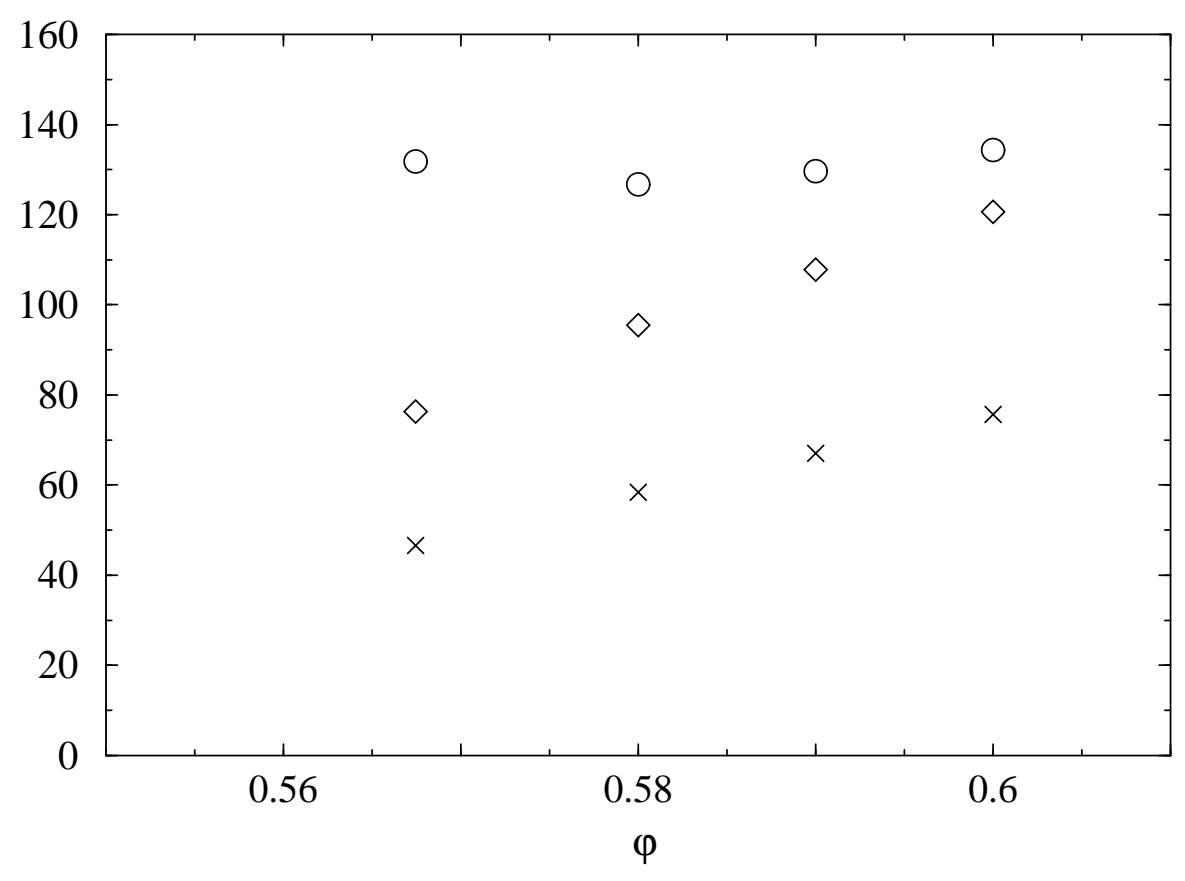

Figure 9: W. Götze and M. R. Mayr 


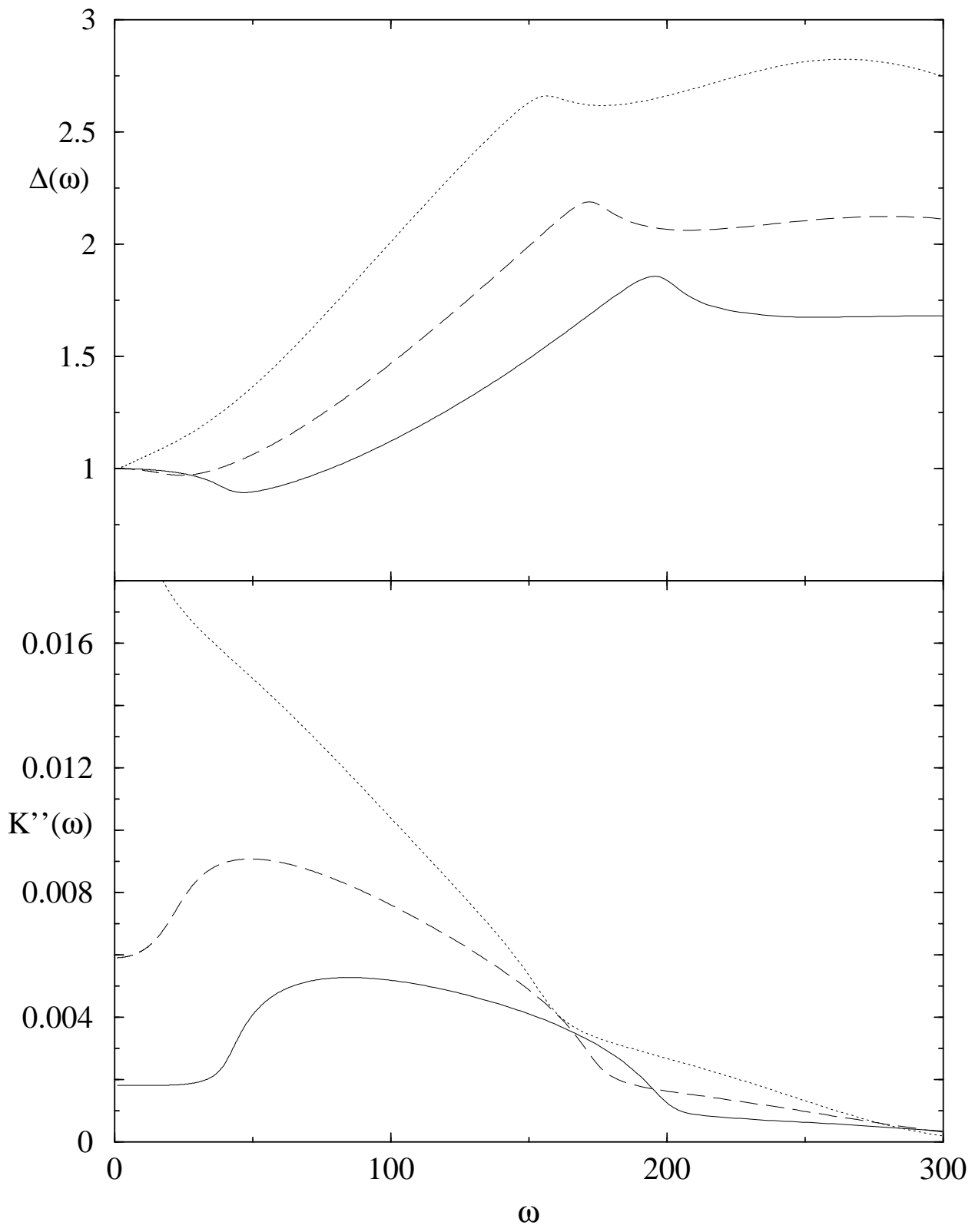

Figure 10: W. Götze and M. R. Mayr 


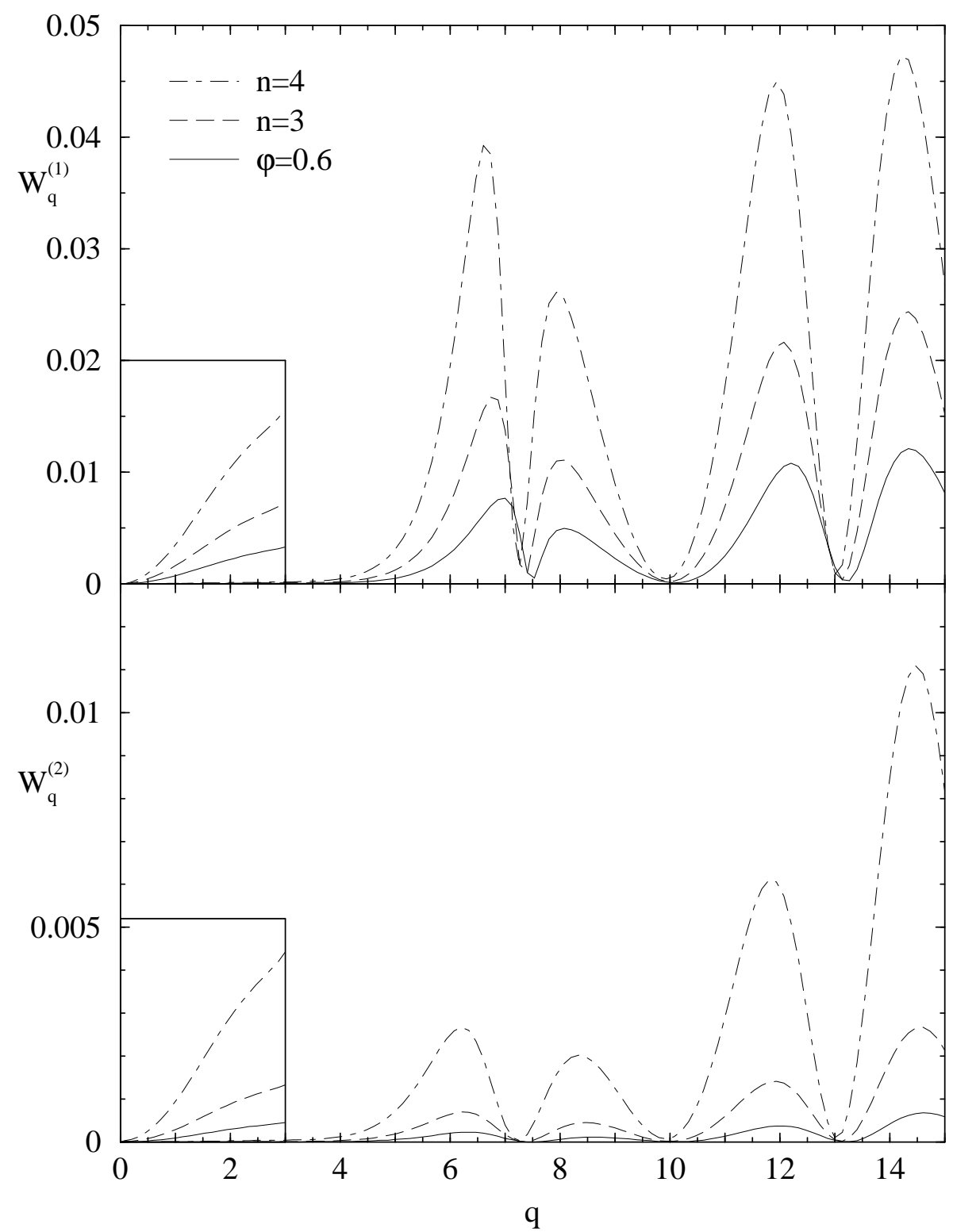

Figure 11: W. Götze and M. R. Mayr 


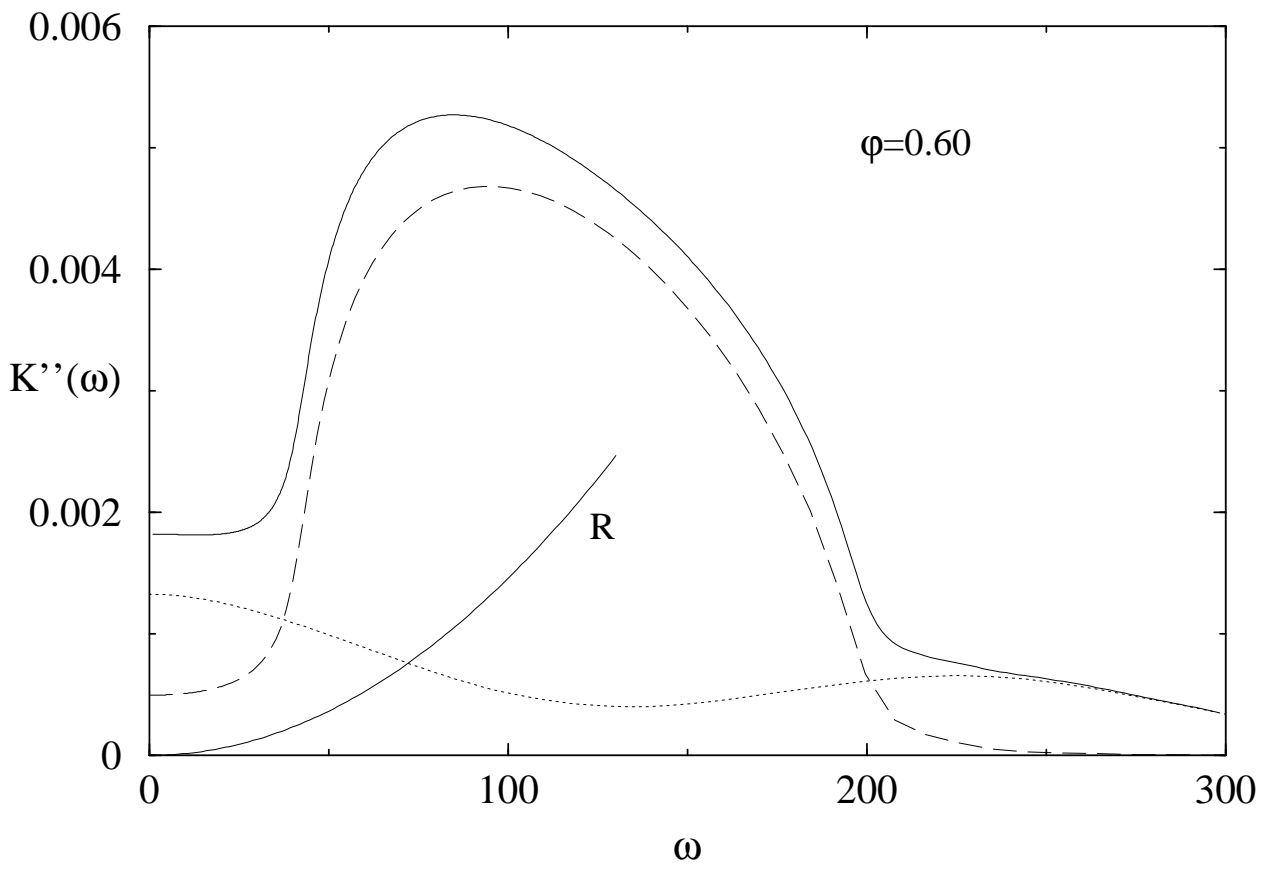

Figure 12: W. Götze and M. R. Mayr 


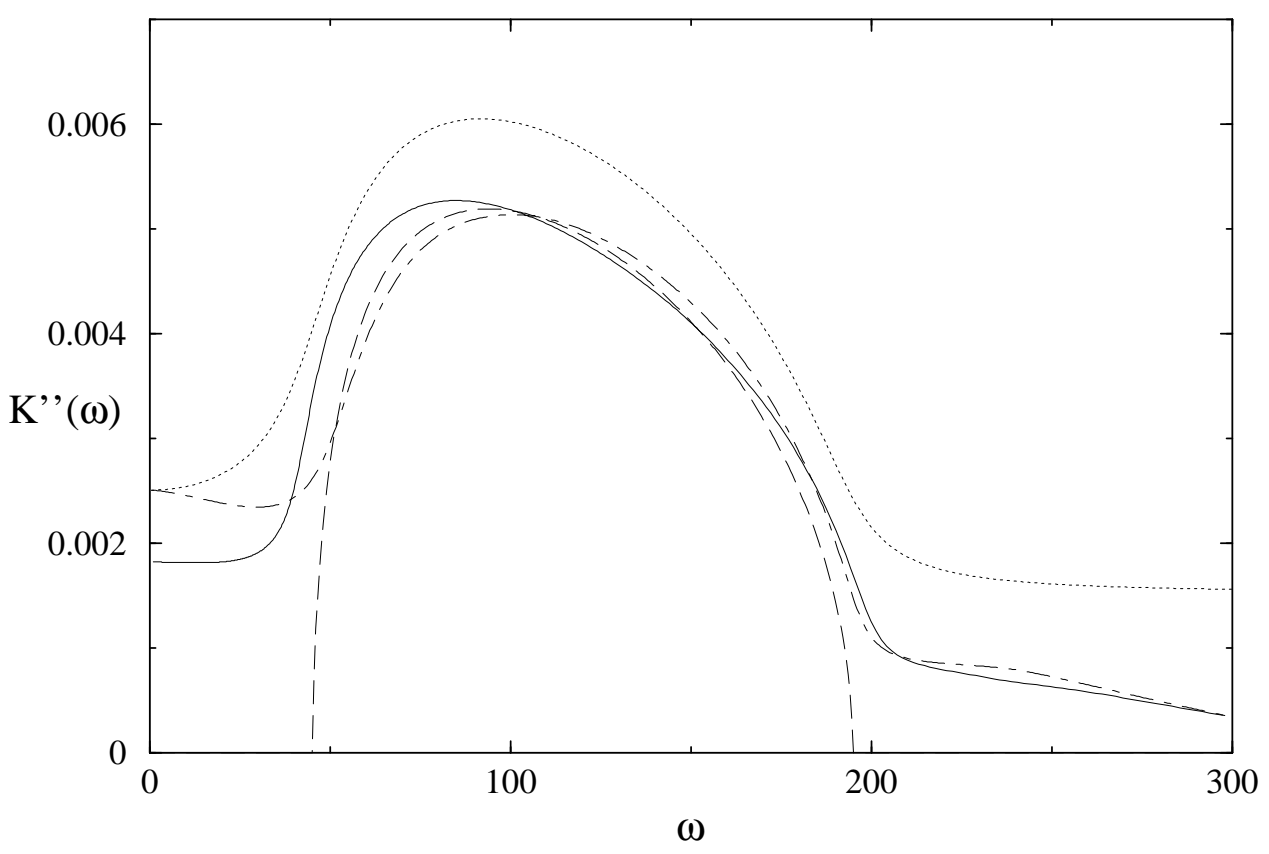

Figure 13: W. Götze and M. R. Mayr

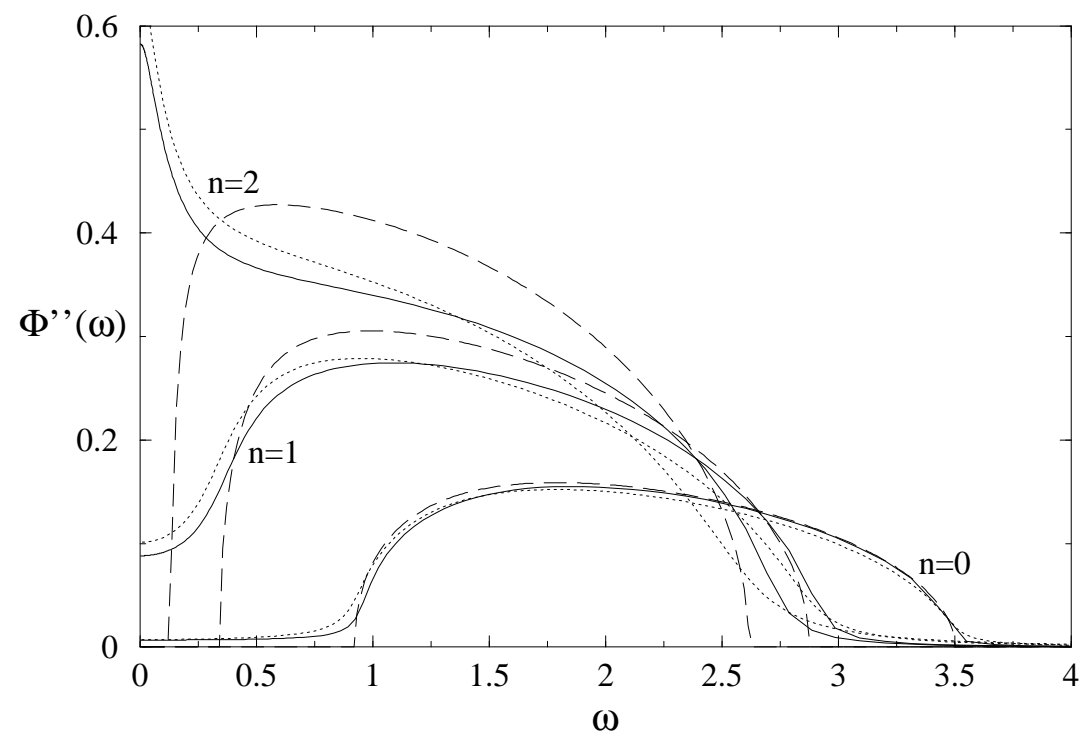

Figure 14: W. Götze and M. R. Mayr 


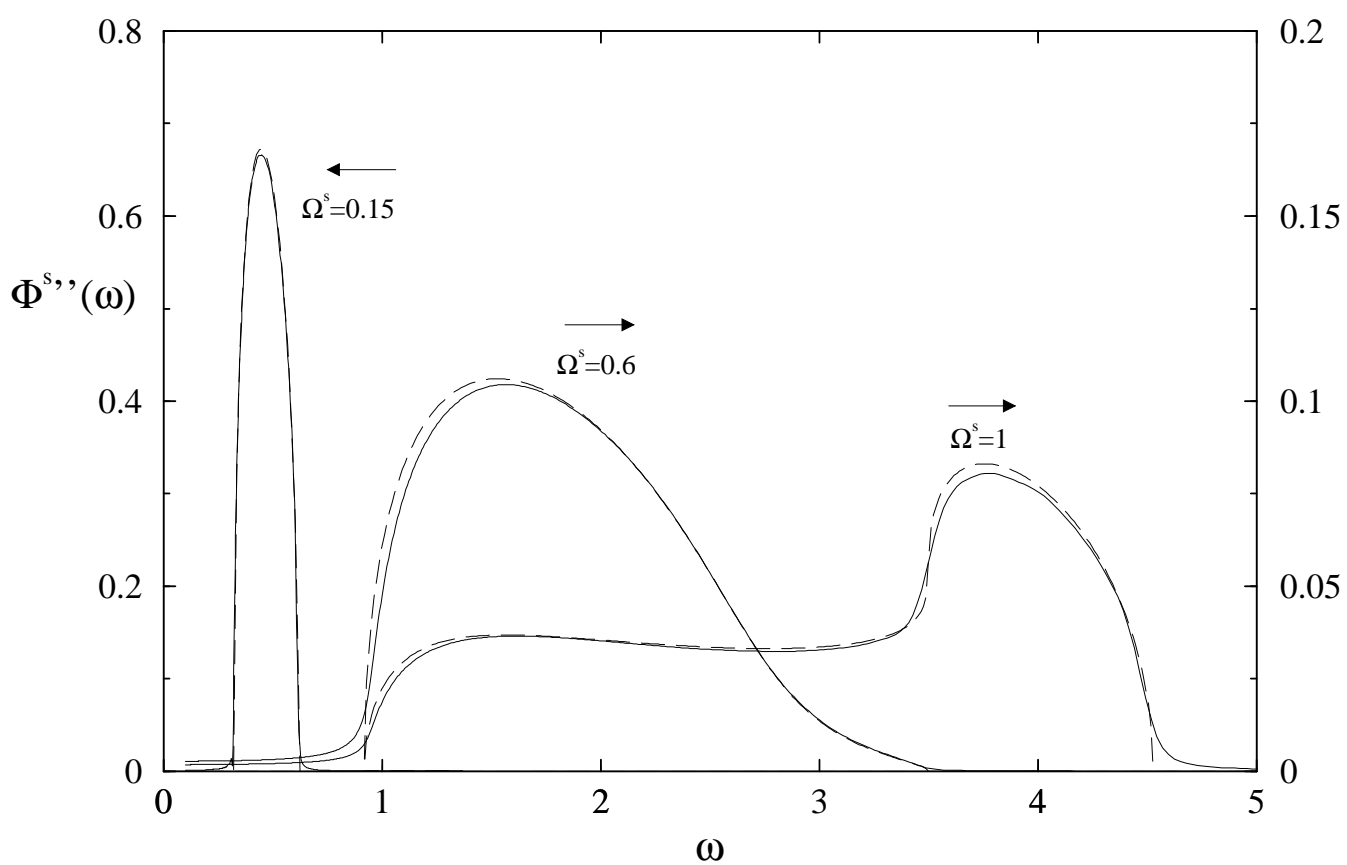

Figure 15: W. Götze and M. R. Mayr 


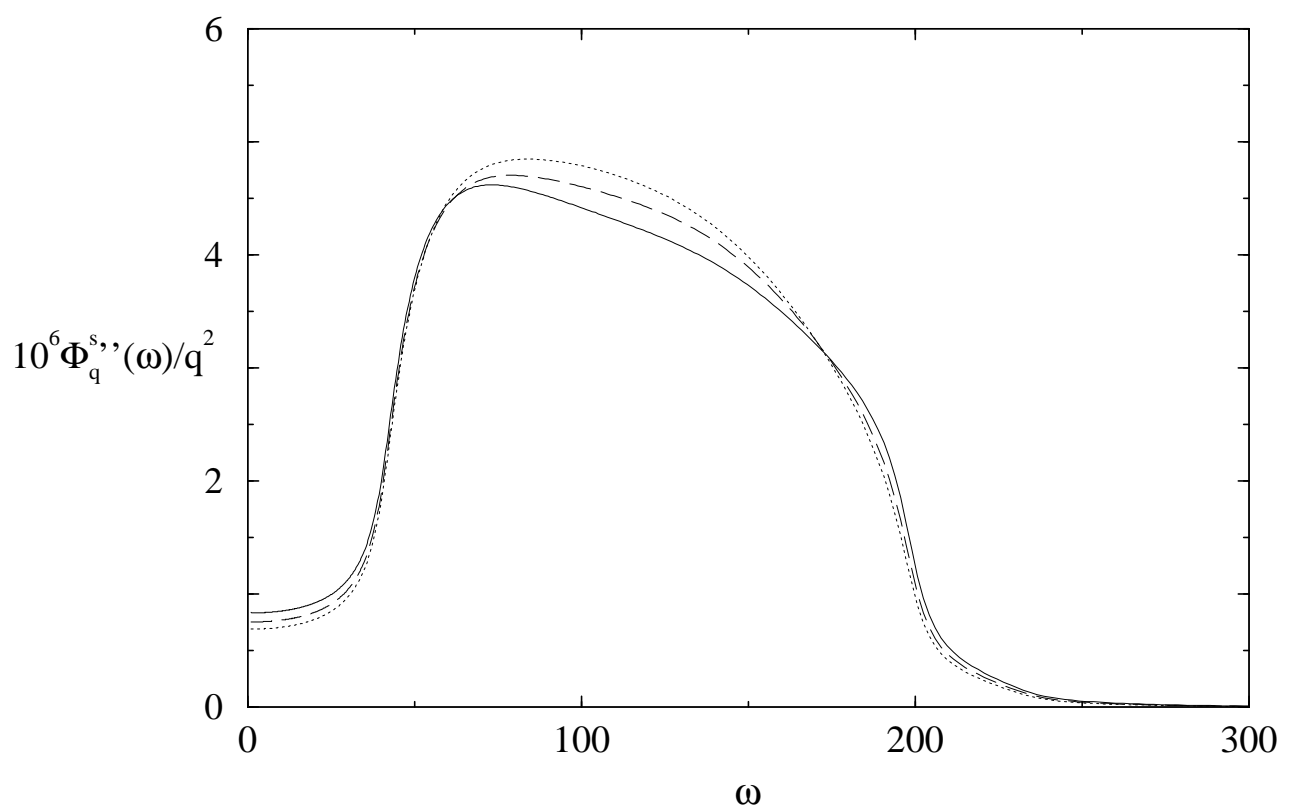

Figure 16: W. Götze and M. R. Mayr 


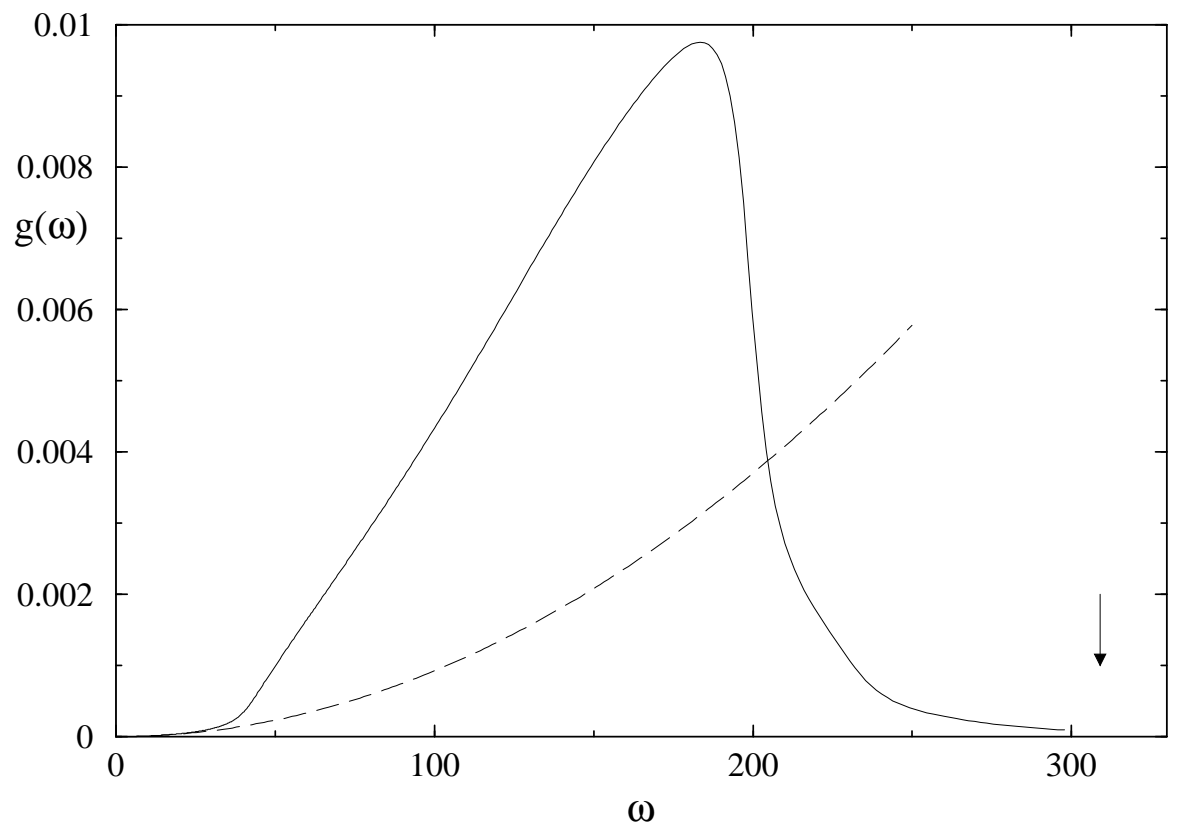

Figure 17: W. Götze and M. R. Mayr 


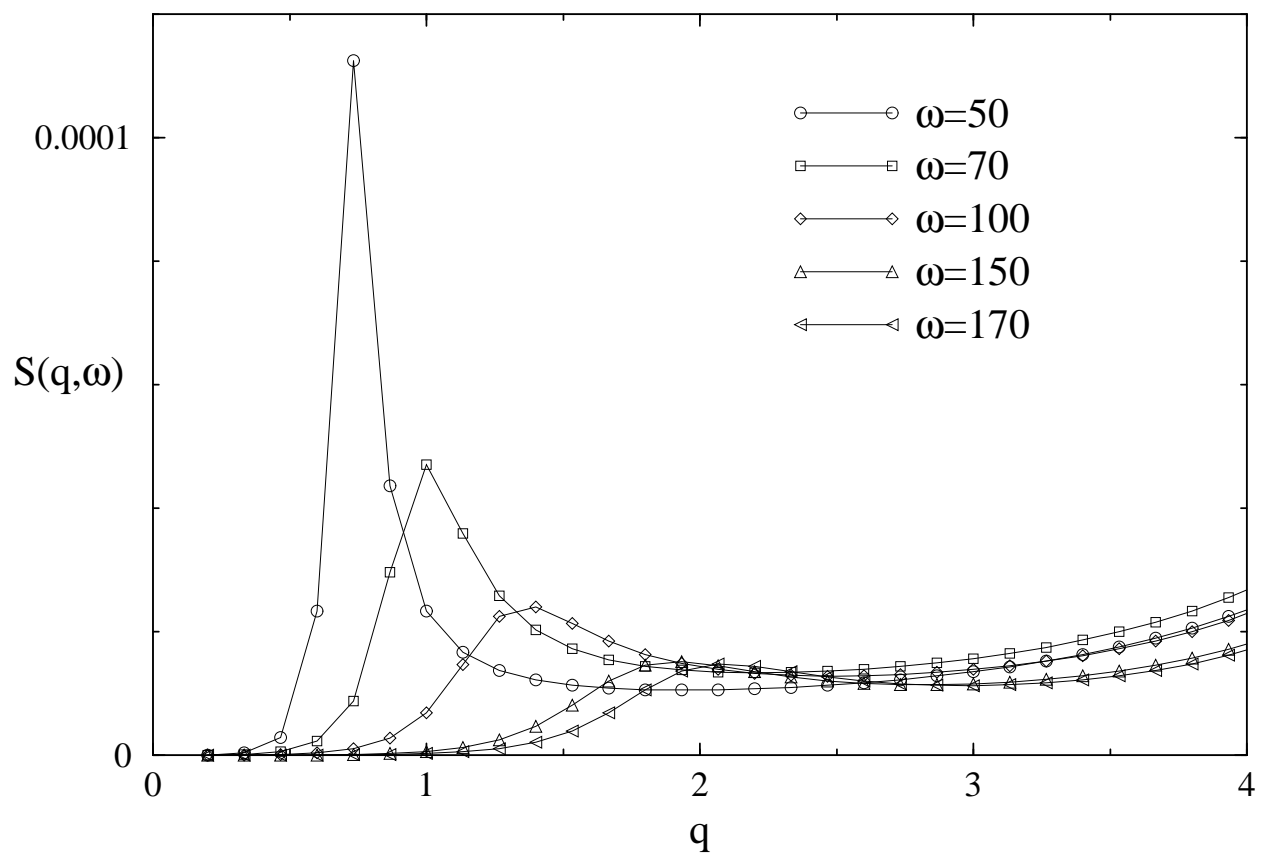

Figure 18: W. Götze and M. R. Mayr 